le portiQue Le Portique

Revue de philosophie et de sciences humaines

31 | 2013

Traduire Heidegger - Lacan / Refonder l'esthétique -

Pierre Kaufmann

\title{
De la traduction à l'interprétation
}

From translation to interpretation

Von Übersetzung bis Interpretation

Jean-Paul Resweber

URL : http://journals.openedition.org/leportique/2677

DOI : 10.4000/leportique.2677

ISSN : $1777-5280$

Éditeur

Association "Les Amis du Portique"

Édition imprimée

Date de publication : 1 novembre 2013

Pagination : 27-71

ISSN : 1283-8594

Référence électronique

Jean-Paul Resweber, « De la traduction à l'interprétation », Le Portique [En ligne], 31 | 2013, mis en ligne le 01 novembre 2015, consulté le 25 mars 2021. URL : http://journals.openedition.org/leportique/2677 ; DOI : https://doi.org/10.4000/leportique.2677

Ce document a été généré automatiquement le 25 mars 2021.

Tous droits réservés 


\title{
De la traduction à l'interprétation
}

\author{
From translation to interpretation \\ Von Übersetzung bis Interpretation
}

Jean-Paul Resweber

\section{Lacan, traducteur de Heidegger}

1 Cette republication se fait en accord avec Jacques Allain Miller au titre du droit moral de l'œuvre de Lacan. Le Portique remercie M. Jacques Allain Miller pour l'accord qu'il a donné à Jean-Paul Resweber.

2 Le texte de la traduction de Lacan reproduit celui de l'article publié dans la revue $L a$ Psychanalyse, 1956, $\mathrm{n}^{\circ} 1$, p.59-71. Le même texte est disponible sur le site www.scrib.com sous le titre Traduction du «Logos » de Lacan. Le commentaire se réfère à la pagination du texte republié dans ce volume.

3 Les citations du texte allemand sont extraites de la conférence Logos, qui, publiée dans Vorträge und Aufsätze, V. Klostermann, 1954 (p. 213-235), présente une seconde version, un peu plus longue, du texte de la conférence publiée en hommage à Hans Jantzen. La traduction d'A. Préau, à la différence de celle de Lacan, prend appui sur cette seconde version qui, à quelques détails et ajouts près, reprend le texte de la première version (Essais et conférences, Le Seuil, « Essais », 1958, p. 249-278).

\section{Introduction}

4 Le texte intitulé "Logos» que publie Heidegger en 1936 est un commentaire du fragment 50 d'Héraclite. Il comporte deux versions: la première est celle d'une conférence qui, prononcée à Brême le 4 Mai 1951, a été publiée dans les «Mélanges offerts à Hans Jantzen » (Festchrift für Hans Jantzen, Berlin, Geb./Mann, 1951), la seconde qui reprend la première, en y ajoutant une digression sur la question de l'oubli de l'Être aggravé par la domination planétaire de la technique, paraît dans le recueil Vorträge und Aufsätze en 1954. Le thème de cette conférence a fait l'objet d'un développement 
dans un cours non publié du semestre d'été sur la Logique (1944). C'est de la première version que J. Lacan publie la traduction, en 1956, dans le premier et unique numéro de la revue La Psychanalyse $\left(\mathrm{n}^{\circ} 1\right)$. Deux ans plus tard, A. Préau présente une traduction de la seconde version dans le recueil Essais et conférences.

Cette traduction originale qui est moins une transcription qu'une transposition du texte allemand dans le texte français, fondée sur « une recherche d'équivalence » et d'assonances, résulte de l'écoute croisée que Heidegger fait du Logos grec et de celle qu'en fait J. Lacan lui-même. Elle est moins littérale que littorale : elle part non de ce qui est dit, du discours écrit, mais du "dire " supposé de Heidegger, même si ce dire est, on s'en doute, infléchi par une lecture inspirée par la psychanalyse: «Quand je parle de Heidegger ou plutôt quand je le traduis, je m'efforce à laisser à la parole qu'il profère sa signifiance souveraine ${ }^{1}{ }^{1}$. C'est ainsi que, entre philosophie et psychanalyse, elle nous découvre un milieu dialogique $\mathrm{d}^{\prime}$ " acheminement vers la parole ", où le sens du texte s'expose sur le mode du «mi-dit». Signalons que le traducteur a envoyé le texte français à l'auteur et que, c'est fort de l'approbation de ce dernier, qu'il l'a publié.

Ce travail de traduction représente pour Lacan une référence majeure, dans la mesure où il met en jeu les interprétations essentielles qui président à sa lecture de Freud: priorité à la question du langage sur celle de l'être, interprétation du réel en termes de processus symbolique, constitution d'un champ de pensée à partir d'une théorie du signifiant qui implique une révision des deux notions cardinales : celles de l'inconscient et de la castration à la lumière des concepts d'absence, de béance, de manque, de signifiant phallique, de vérité et d'Autre. Dans son séminaire sur L'Éthique de la psychanalyse, qu'il dispense quatre ans après avoir rédigé sa traduction, Lacan revient fièrement sur ce travail, à l'occasion d'un commentaire du célèbre texte de l'Antigone de Sophocle, faisant l'éloge du « monstre » humain :

Il y a pas mal de choses formidables dans le monde, mais il n'y a rien de plus formidable que l'homme,

en rapportant le "mécanisme » de l'ingéniosité de l'homme qui, par ses inventions, s'emploie à conjurer la mort, au pouvoir du Logos qui lui donne mandat (sophon) pour cette tâche : "Il y a là quelque chose de sophon qui n'est pas si simple. Je vous prie de vous rappeler, dans le texte de Heidegger que j'ai traduit moi-même pour le premier numéro de la Psychanalyse, l'analyse du sens héraclitéen de sophon, sage, et de homologein, dire la même chose. Ce sophon a encore toute sa verdeur primitive. Il y a quelque chose de sophos dans le mécanisme, mêchanoen. Il y a quelque chose hyper elpid'echôn qui va au-delà de tout espoir, et qui erchei (en français: devance en transitant). C'est cela qui le dirige tantôt vers le mal, tantôt vers le bien. C'est dire que ce pouvoir, ce mandat - c'est ainsi que je traduis sophos dans l'article dont je vous parlede ce qui est déféré à lui par ce bien, est éminemment ambigu » ${ }^{2}$. De l'aveu de Lacan, le texte traduit se présente comme un "article» : il articule des lieux signifiants (topoi) tantôt explicitement convoqués, tantôt implicitement sur le mode de l'allusion, de l'évocation ou de la citation, lorsque, notamment, Lacan se réfère aux notions de parole et de vérité, de langue et de langage, de réel et de symbolique. Mais il faut de suite souligner que ce qui, dans ce texte, "articule » ces notions, c'est la question de l'Un héraclitéen que Lacan ne cesse de « reposer » et de « retraduire » tout au long de ses écrits.

7 Le commentaire qui suit n'a d'autre ambition que de démontrer l'ingéniosité linguistique et l'originalité sémantique de la traduction. La première partie de ce 
commentaire s'emploiera à mettre en perspective les configurations signifiantes du texte induites par la traduction lacanienne. La seconde partie tentera de montrer que cette traduction cherche à rendre compatibles la pensée de l'Être et la théorie de l'inconscient.

8 On le sait, le concept est souvent le déploiement d'une métaphore refoulée et la métaphore est souvent, quant à elle, la cristallisation ou la condensation (Verdichtung) d'un geste (Gebärde). Le chemin de la pensée conduit à l'invention de ce geste, c'est-àdire à sa découverte et à sa reconstitution. Toute langue est en gestation de concepts et le philosophe qui se laisse surprendre par l'écoute des métaphores de la langue, n'a de cesse de les entendre, pour tenter d'en extraire des lignes de pensée et de vie. Le langage de l'étonnement philosophique (thaumazein) n'est pas celui de l'herméneutique qui s'épuise en «reprises ", mais celui de la maïeutique, qui fait naître la surprise. Le philosophe et le psychanalyste partagent le plan de travail d'une langue en gésine, comme dit le commentaire, d'une langue tierce dont le grec est la figure emblématique et dont on ne finit pas d'explorer le trésor des signifiants qu'elle recèle.

\section{Les motifs de la traduction}

9 La traduction de Lacan poursuit deux objectifs : d'une part, celui de retourner au sens grec du Logos à partir d'une écoute des mots de la langue allemande, conduite par Heidegger, mais aussi d'une écoute des mots de la langue française, qui partagent avec ces derniers une proximité homophonique, sinon étymologique; d'autre part, celui de faire saillir la vérité de l'entrechoc musical des mots, en produisant des effets singuliers d'écriture induisant des significations inédites dues au jeu des équivalences, des connotations, des assonances ou des consonances. Chez Lacan, comme chez Heidegger, nous trouvons une démarche de pensée similaire qui s'emploie à concasser la langue ordinaire, pour faire surgir une autre langue où le sens se fait entendre au travers des glissements discontinus des mots. Tel est le travail de décryptage, auquel se livre Heidegger en retournant aux Présocratiques, tel est aussi celui que poursuit Lacan en revenant au texte de Freud.

10 1. La méditation heideggérienne du Logos a exercé sur Lacan une durable influence qui tient certes à la féconde polysémie des termes, mais aussi au double jeu de contradictions dont elle s'entretient. Logos signifie, en effet, calcul, discours, sens et le verbe $\lambda \varepsilon ́ \gamma \varepsilon ı v$ auquel il renvoie signifie parler, dire, nommer. Il renferme en lui-même une première contradiction que lève le fragment d'Héraclite: celle qui existe entre l'explicitation d'un sens énigmatique issu du dévoilement de la vérité et la formulation discursive de ce sens en termes de processus logiques et rationnels. La tension entre ces deux pôles prend alors la forme d'un premier paradoxe qui ne peut qu'interpeller Lacan : le Logos interprété dans le langage du discours occulte le rapport à l'excès d'une vérité auquel il donne pourtant accès.

11 Ce premier paradoxe se trouve relativisé, dès lors qu'il est éclairé par la partition du voilement et du dévoilement qui caractérise l'événement de la vérité et que Lacan déplace sur le double champ de l'inconscient et du conscient. On comprend aisément que le Logos soit à la fois le chemin privilégié qui ouvre la porte de l'inconscient et comme il l'insinue au début du texte, le barrage qui lui fait obstacle. Car la parole surgit, à la faveur de ces trébuchements de l'énonciation que sont le malentendu, le mot d'esprit, le lapsus, le silence ou bien encore le jeu de mots, faisant effraction dans 
l'énoncé sur le mode du mi-dit. Ainsi fait-elle barrage au Logos, qui, lui-même «barré », se présente sur le mode du retrait. Le Logos « est aussi lockos, l'embûche où quelque chose est relégué sous ce qui est allégué " ${ }^{3}$.

2. La seconde contradiction héraclitéenne nous est plus familière: elle met en conflit le mouvement et le repos, l'être et le devenir. Héraclite nous rappelle à l'ordre des contraires: le fleuve, en coulant, ne cesse de différer son cours, sans pour autant devenir autre que lui-même. Le cours du fleuve rassemble la diversité dans l'unité, étale la durée dans les variations du mouvement qui battent le rythme du temps. Tout en étant sculpté par les aléas de l'écoulement, le lit du fleuve ne reste-il pas le même ? Le terme lais, vieux mot français, qui sera remplacé au XVII e siècle par le mot legs, exprime, selon Lacan, le mode de transmission héréditaire du Logos, mais il désigne aussi le lit laissé par les eaux qui se retirent, une fois accompli leur travail d'érosion. Les métaphores de la traduction lacanienne sont hantées par la vision héraclitéenne de l'écoulement de toutes choses. Le Logos rassemble l'être et la multitude générée par le devenir, il met en tension les forces antagonistes, il nous enseigne que c'est le contraste qui est le moteur de l'émergence d'un monde structuré et nous enjoint de nous approprier ces contradictions pour exister comme être-au-monde : comme " parlêtre ».

Cette double partition héraclitéenne est énoncée dans des jeux de langage qui engagent des jeux de vie. Le rapport existant entre le sens et l'énoncé, comme celui existant entre l'être et le mouvement, se décline en une cascade de contradictions qui procèdent des jeux d'occultation et de dés-occultation qui président à l'expérience de la vérité. Mais ce sont ces alternances qui créent la surprise : celle de durer, d'exister, de devenir. Or, c'est bien le Logos qui est le mandataire d'une telle surprise : toutes choses tiennent en l'Un et de l'Un. Il n'y a pas de résolution harmonieuse des contraires, comme le préconise Platon qui nous propose une lecture musicalement «ordonnée » de la pensée d'Héraclite: «L'un coïncide... en différant de lui-même, comme c'est le cas pour l'harmonie de l'arc et celle de la lyre " (Le Banquet 187a-b). Le Logos tient ensemble le sens et le discours, l'identité et la différence : il nous prévient que c'est la contradiction qui nous fait vivre, espérer et croire. Et le lieu de résolution de la contradiction, c'est l'inconscient, l'Un/Bewusst, l'une/bévue, comme aime à en rajouter Lacan.

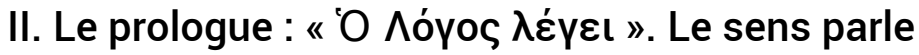

14 Venons-en à la célèbre parole d'Héraclite (fragment 50). En voici «la traduction courante » reprise au vol par Heidegger et retranscrite en français par A. Préau : «Si ce n'est pas moi, mais le Sens, que vous avez entendu, il est sage alors de dire dans le même sens : Tout est Un " ${ }^{4}$. Lacan la fait sienne provisoirement, mais il la rectifie aussitôt en revenant à la lettre du texte grec qui "pointe " la place du locuteur, en l'occurrence celle d'Héraclite, désigné non au nominatif (ce n'est pas moi), mais au génitif (ce n'est pas de moi) et qui, de surcroît, récuse la formulation commune qui ferait de l'Un un attribut du Tout (Tout est Un), pour mettre en lumière la priorité logique de l'Un (l'Un est Tout) : «Si ce que vous avez entendu n'est pas de moi, mais du sens, Il est sage aussi de dire pareillement à ce sens: l'Un est Toutes Choses " ${ }^{5}$. Ce double avertissement nous rappelle que Héraclite ne prononce pas cette parole en son nom propre, puisque, d'une part, le moi dont il fait état est déjà inscrit dans le champ du sens, en l'occurrence celui du Logos et que, d'autre part, si l'Un se pose comme un 
pluriel singulier, c'est avant tout parce qu'il est un singulier pluriel, unissant toutes les choses sans jamais les totaliser (Eins ist alles).

Ce qu'est le Logos, nous le recevons de son legein, des effets qu'il produit en l'homme, en se dispensant. Or, le verbe legein signifie dire, parler, raconter. Mais ce sens prévalent est articulé à un autre sens dont témoigne l'étymologie : Lacan le rend par le verbe homophone léguer qui croise les trois champs sémantiques : celui du legein grec, celui du legen allemand et celui du legere latin. Le verbe latin a le sens de rassembler ce que l'on ramasse, le verbe allemand de mettre et de poser, le verbe grec qui comporte l'acception la plus large met l'accent sur le geste de déposer, comme l'indique le verbe legein usité à la voie moyenne, intermédiaire entre l'actif et le passif, mais aussi sur les geste de coucher et de réserver comme le suggère les substantifs qui en dérivent: leckos, le lit du repos et lockos, l'embûche « où quelque chose est relégué sous ce qui est allégué » (61). Mais c'est le verbe allemand legen, mettre et poser, qui, conjoint au verbe grec legein, coucher, va fournir à Lacan la première articulation signifiante : legein signifie «mettre à reposer » le sens qu'est le Logos.

Mais par le jeu de la traduction, legein, legere et legen, Lacan raffle la mise en condensant le sens des termes grecs (legein), latin (legere) et allemand (legen) dans le verbe français léguer. La suite de la traduction confirme la place de ce signifiant maître : le sens comme legs est relégué, allégué, délégué. Il nous renvoie au signifiant élidé, la loi (lex) dont il dérive à l'accusatif (legem). Lacan rapproche astucieusement le mot legs du mot lais qui l'a remplacé au XVII ${ }^{e}$ siècle, en dépit de toute filiation philologique, mais qui, issu du verbe laisser, nous renvoie au champ sémantique de mettre, poser et déposer, coucher, mais aussi au champ sémantique du dépôt et du propos, des traces et du récit, puisque le lais désigne aussi bien la plage raviné par le retrait des eaux que le poème et la chanson de geste.

Legein signifie recueillir les sens comme un legs ou comme un lais et le mettre-àreposer, le mettre-à-gésir. À la suite de Heidegger, Lacan moissonne les signifiants, en suivant pas le fil de la métaphorique de la récolte : collecter, c'est colliger : présenter ensemble (cum-legere) et mettre-à-reposer, c'est recueillir ce que l'on a cueilli. Heidegger décrit les gestes qui président à la cueillette, pour souligner que chaque séquence des activités en cours est finalisée par la mise à l'abri, la mise au repos et la mise en dépôt, la mise au gîte, la mise en réserve : autant d'expressions qui soulignent que c'est le recueillement de la cueillette qui est déterminant. La moisson est un cadeau du ciel qui s'offre aux mains qui ramassent et émondent et ces mains tendues et ouvertes dessinent les gerbes et les grappes choisies pour être sauvegardée : «Cet "enplus" (jenes mehr) qui dans la moisson dépasse la rafle qui s'en empare, ne vient pas seulement s'y ajouter ». Ce qui se présente dans le legein est retenu, parce qu'il nous est réservé et " nous concerne et par conséquent nous regarde ». Retenu et, d'une certaine façon, barré : Lacan introduit en parenthèses une phrase de la seconde édition du texte qui lui paraît significative: "Legi » veut dire en alémanique le barrage qui dans le courant se dresse au-devant : du torrent liquide ».

Le legein couche et met en dormition ce qu'il présente: il fait son lit dans l'hypokeimenon, neutre du participe passé du verbe passif keisthai qui signifie être répandu, gésir. C'est ce terme qui a désigné le sujet grammatical, la substance, puis la subjectivité. Heidegger y voit le signifiant de l'émergence de "l'être de la présence de ce qui se présente dans le dévoilement». La traduction de Lacan tend à lui conférer une touche subjective, en insistant sur l'implication de l'homme dans le legein : « Le keisthai, 
le pour-soi de la présentation de ce qui est ainsi relégué, du hypokeimenon n'est ni plus ni moins que l'être de la présence de ce qui se présente dans le dévoilement. Dans ce legein du hypokeimenon, le legein du choix et de la collation demeure serti». Lacan rappelle en note que le terme grec a son équivalent latin dans le terme subjectum, ce qui gît dessous, le sujet. C'est là, Da-sein, que le Logos, le sens, est présent : dévoilé dans le jeu de son voilement, allégué dans sa relégation même.

Reste la question de savoir comment on passe de cette présentation reposante ou, comme traduit A. Préau, de cette Pose accueillante au sens qui est celui de dire et de proférer. Heidegger nous rappelle que c'est la prédominance de ce sens usuel qui nous a fait oublier le sens primordial de legein. Il précise que c'est le legein lui-même qui se laisse surmonter par un aspect en lui prédominant: celui de dire et de proférer. Mais coupée "l'empreinte originelle qui est l'essence du langage", la parole proférée se trouve privée de son efficacité symbolique et réduite à une expression vocale et à une signification, disons au couplage du signifiant et du signifié. Car le legein ne comporte pas deux acceptions, mettre-à-reposer et dire, dont la première serait reléguée au profit de la seconde. C'est bien dans le dire, c'est bien dans la parole proférée que se réalise la présentation du sens, le dévoilement de la présence. Parler, c'est s'accorder à l'événement du legein, en le faisant advenir comme tel.

20 Si une telle parole nous semble, malgré tout, aller de soi, n'est-ce pas parce que nous l'avons depuis toujours apprivoisée au sein de la glose métaphysique et théologique, en déclinant le Logos dans l'éventail rationnel de l'énoncé, de la loi, de l'ordre du monde ou de la causalité divine? Certes, on ne saurait renier le sens premier que comportent, dans la langue grecque, les termes affiliés au Logos: le verbe legein signifie dire et parler, le participe passé legomenon définit le contenu de ce qui est énoncé et le substantif logos caractérise l'énoncé. Mais le fragment d'Héraclite doit être « entendu » en un sens plus fondamental : il nous enjoint d'oür (akouein) le Logos, de nous livrer, comme locuteurs (emou, legontos) à son écoute, car il est le dire et le dit à la fois, l'énonciation et l'énoncé : l'événement qui fait corps avec son incarnation dans le «parlêtre » (homologein).

\section{L'héritage : le legs (legen) et le lais (die Lege)}

21 À titre de prologue, Heidegger questionne l'énigme (Rätsel) du dire originel. Il en dégage une détermination primordiale, en partant du champ sémantique du verbe grec legein, dont il examine les concrétions métaphoriques qui dessinent des gestes oubliés. Le Logos, c'est-à-dire le Sens, se pose et se repose en se rassemblant. J. Lacan a sans doute été séduit par le jeu des associations homophoniques et étymologiques qui font glisser le sens en déployant les harmoniques du «soit-disant" radical leg: le verbe legein, qui explicite le geste testamentaire du Logos nous renvoie au verbe latin legere qui signifie rassembler et lire, mais, du même coup, au verbe allemand legen qui signifie poser. À entendre ces signifiants qui modulent et modalisent le sens, en les articulant les uns aux autres, on entend curieusement résonner le signifiant élidé inscrit dans le radical «leg»: recueillir, déposer et rassembler. C'est l'instance de ce dernier dans la lettre du radical que Lacan, fidèle aux jeux des associations, rend par le terme de legs qui évoque à la fois le geste de rassembler par testament, de lire ce qui est transmis, de transmettre ce qui se donne à lire. La lecture n'implique-t-elle un geste de collecte (colligere) des signifiants dans l'acte même de la saisie globale par laquelle elle s'empare de l'écrit? 
Mais le jeu des associations ne saurait s'arrêter là. Si l'on parcourt le champ sémantique ouvert en continuant d'y glaner les signifiants, on découvre que le verbe legein se conjugue aussi dans une voie intermédiaire entre l'actif et le passif, appelée voie moyenne et que Lacan se plaît à désigner de l'ancien mot "déponent» (deponere en latin signifie déposer): la conjugaison passive de ce verbe à la forme active nous renvoie aux termes précités de lekhos, lit du repos et lockos, guet-apens, qui nous rappellent que legs du Logos s'allègue, se délègue et se relègue. Lacan ne cesse, par les jeux de la traduction, de traquer le signifiant enfoui dans l'écrin du radical. Cette nouvelle exploration nous suggère que le dépôt du legs qui rassemble la transmission en la «couchant» par écrit est une mise au repos. Telle est la nouvelle séquence signifiante induite par la voie moyenne ou par le « déponent » du verbe grec legein que Lacan condense dans le mot « lais » qui, comme ce dernier, ne provient pas du mot loi même si il a eu ce sens à l'origine -, comme le précise en note le traducteur, mais du mot «laisser». Un tel signifiant exprime avec bonheur l'idée de coucher et l'idée d'étendre, "de porter à gésir » (zum liegen bringen), comme l'énonce judicieusement Lacan ${ }^{6}$, car le lais, mais la traduction n'exploite ce sens que de façon oblique, désigne aussi, on l'a déjà noté, l'espace ouvert par le retrait des eaux : le littoral, le lit des eaux, la grève. Rassembler (versammeln), c'est aussi étendre (ausdehnen).

\section{La moisson (die Lese) : cueillir (lesen) et recueillir (legen), lier et lire}

Lacan finit par traduire le geste du Logos, le legein, par "mettre à reposer ", alors que Préau se satisfait de le rendre par le verbe "étendre». Mais cette logique signifiante, celle de rassembler, étendre ou coucher, se cristallise dans la métaphore de la moisson. Nous voilà subrepticement passés du verbe grec legein au verbe allemand legen, poser, en faisant un rapide détour par le verbe latin legere, rassembler et lire et, au final, nous avons glissé du verbe allemand legen, poser, à cet autre verbe, lesen, qui signifie récolter et lire: "Jedes Lesen ist schon Legen. Alles Legen ist von sich her lesend" ${ }^{7}$. Ce que Préau traduit sobrement: "Cueillir, c'est toujours étendre. Étendre, de soi-même, est toujours cueillir " 8 . Lacan, de son côté, rattache d'emblée le double geste de " cueillir/ recueillir " à celui de la lecture. Quiconque lit, cueille le sens, le glane et l'émonde, mais, du même coup, il le recueille, le met en dormition, en attendant de le réveiller à la faveur d'une autre cueillette. Voici la formulation de Lacan qui dessine en quelque sorte le phrasé de l'inconscient : «Ce qui s'y lit est déjà lit de la reposée, et tout lit de la reposée est de soi-même ce qui se lit dans ce qu'on collige " ${ }^{9}$.

À la différence de Préau, Lacan ne suit pas Heidegger, lorsqu'il rapproche le verbe latin legere, qui signifie rassembler du verbe allemand lesen qui signifie ramasser et réunir. Il passe sous silence ce glissement du latin à l'allemand souligné par Heidegger. En commettant cette omission, qui n'a rien d'un lapsus calami, Lacan entend relativiser l'usage de la référence dominante et problématique que Heidegger fait de la langue allemande et mettre en valeur le fait que la langue française est, aussi bien que l'allemand et le grec, en mesure de faire entendre au lecteur et au locuteur, le Logos qui parle en elle. Telle est la trouvaille de Lacan : traduire le verbe grec legein et le verbe allemand legen par le verbe français léguer qui implique le double sens de déposer et de proposer.

Revenons sur ces deux signifiants - charnières : le legs et le lais. Le premier vient du latin legem, accusatif du mot lex qui signifie loi. Le second, usité au XII ${ }^{\mathrm{e}}$ et $\mathrm{XIII}^{\mathrm{e}}$ siècles (lais ou laisse) s'est substitué au mot legs, sans pour autant entretenir avec lui une 
quelconque parenté étymologique. Lacan le convie pour désigner le Logos qui nous est laissé en héritage, mais il n'est pas sans savoir qu'il désigne aussi non seulement l'espace laissé par le retrait des eaux, mais aussi les écrits poétiques laissés en testament par un auteur, les dits, les récits de ce dernier ou les couplets d'une chanson de geste. En prenant appui sur ces significations, il fait l'invention, dans la langue française, d'un champ sémantique inédit, capable de rendre aussi bien compte du legein grec que du legen allemand : léguer, legs, loi, lais... Le lais fait le lit du langage, se dépose dans l'écriture du récit ou du poème, lie en " gerbes » les traces, qui sont les traits ou les marques de retraits successifs, pour les offrir à lire.

Nous voici parvenus au terme de la première séquence du commentaire : le verbe latin legere et le verbe allemand lesen signifient d'abord rassembler et ramasser, mais aussi lire, voire, si l'on file l'anagramme, lier. Lire, et c'est bien ce que fait Heidegger dans son commentaire et Lacan dans sa traduction, c'est moissonner les signifiants. Mais le geste de la moisson ouvre une autre séquence qui déplace et dépasse le sens de la première, car il inclut non seulement la collecte sélective des fruits, mais aussi leur mise à l'abri et leur sauvegarde : il vise à cueillir et donc à choisir, à accueillir (sammeln) et à recueillir en rassemblant (versammeln). Mais, précise Heidegger, le sens d'un tel geste dont le Logos est porteur ne se réduit pas à l'enchaînement de ces diverses activités : celle de la cueillette par émondage (Ablesen) ou par assemblage (Erlesen), celle du rassemblement des fruits et celle de l'engrangement de la récolte. Car le travail de la moisson, dès son point de départ et durant son exécution, mais, bien sûr, lors de son accomplissement, est finalisé par le geste de la sauvegarde (bergen) et de la mise à l'abri (Hut). Il convient, par conséquent, de ne pas se laisser aveugler par l'ordre manifeste, qui, dicté par les étapes du travail, dissimule l'ordre constitué par les traits (Züge) signifiants qui modulent en contrepoint le sens même de la récolte.

On le voit, le langage de la moisson ne se limite pas à l'enchainement métonymique de tâches qui se substituent les unes aux autres : il est, du début à la fin, inspiré par l'acte de sauvegarder. C'est cet acte-là, jenes mehr, cet en-plus de la récolte, que s'empresse de pointer Lacan, qui marque à la fois la différence spécifique des tâches et leur unité générique. Cet en-plus est l'hyperbole d'où tirent leur sens tous les gestes symboliques de la moisson : «Moissonner est pourtant plus que le pur fait d'amonceler. Il fait partie de la moisson de rentrer la récolte. C'est ici le ressort de sa mise à l'abri, qui lui-même relève de sa mise en réserve. Cet «en-plus » qui dans la moisson dépasse la rafle qui s'en empare, ne vient pas seulement s'y ajouter. Il n'en est pas différent de l'entrée en jeu du moment où elle se ferme. La mise en réserve de ce qui doit être rentré a déjà marqué sa prise sur les démarches de la moisson dès leur départ et sur leur ensemble dans l'intrication de leur suite ${ }^{10}$.

Mais, on s'en doute, la sauvegarde qui est mise à l'abri ou mise en réserve s'accomplit dans un espace : celui d'un lit, d'un site (Lage) ou d'un gîte. Là où Préau parle de mettre en réserve, Lacan parle de porter au gite. Il convient, pour mieux saisir le mouvement de la présentation du Logos, d'inclure, dans le geste du «legen », celui de la mise à l'abri. Ainsi, le legs ou le lais devient le lieu, où l'on met-au-repos, où l'on met-à-gésir (zum Liegen bringen) le sens. Le sens se pose dans le legs, se dépose dans le lais, où il s'offre à lire parce qu'il nous concerne: dass uns das Vorliegende anliegt und deshalb angeht ${ }^{11}$. Si le legein du Logos signifie: mettre à reposer, déposer, le Logos comme tel se présente, selon l'expression étincelante de Lacan, comme "le lais où se lit ce qui s'élit " ${ }^{12}$. Telle est la traduction ou plutôt la lecture que fait Lacan de la formule de Heidegger, die lesende 
Lege, qui lie le geste de la pose, voire celui de la pause, à celui de la récolte, die Lese. A. Préau, fidèle à la littéralité, parle de "Pose accueillante " ${ }^{13}$. Lacan cisèle l'expression, de manière à ce que celle-ci puisse, en condensant les associations signifiantes précédentes, suggérer le travail moi-sonneur de l'inconscient.

Les trouvailles de Lacan rendent au mieux compte de la pensée de Heidegger. Comme tel, le legein est un legs qui fait lais, mot qui évoque l'idée d'une transition ouvrant l'espace d'un tracé symbolique (laisser passer l'héritage, le prendre en charge et le mettre en garde). Ainsi, se présente-t-il sous les divers modes de l'allégation (vor-lesen) : mise au repos (niederlegen) ou assignation, mise au dépôt ou relégation (hinterlegen), mise à l'écart ou consignation (zurücklegen). Il élit domicile dans le lais en s'y « excrivant », il y fait son lit, il s'y élit en s'y donnant à lire. Les connotations de ce vieux mot français n'ont pas échappé au traducteur. Le lais, c'est bien de l'écriture : celle du codicille testamentaire, celle du tracé laissé par les eaux enfuies, celle du traçage symbolique de la narration poétique. Retenons le message : le Logos se présente comme une écriture primordiale qui rassemble, en les mettant à reposer, les éléments multiples qu'elle convoque : «En quoi mettre-à-reposer veut dire aussi bien mettre-àreposer l'un contre l'autre, soit mettre ensemble à reposer. Mettre en ce lit est donner à lire. La lecture qui nous est la plus connue, à savoir celle d'un écrit, reste, si prégnante qu'elle soit, n'être qu'un des modes du colliger, au sens de ramasser-en-présentant " ${ }^{14}$.

La métaphore de la moisson comme collecte, rassemblement et sauvegarde est le signifiant-maître : elle commande l'interprétation du Logos comme legs, lais et lecture. Si le « lire " est un mode transcendantal du legein lui-même, il fonde notre conception même de la lecture. Lire, c'est certes épeler les mots, un à un, mais c'est aussi les lier, les rassembler dans des configurations signifiantes et surtout les sauvegarder: les «mettre-à-reposer ", les consigner (cum-signare). À sa manière, le lecteur recompose "le lit où se lit ce qui s'élit ». Il extrait le sens du lit de la reposée et l'y reconduit. Ainsi se déroule la spirale de la lecture : on lit ce qui est mis à reposer et on met à reposer ce que l'on lit. Heidegger renverse l'ordre commun qui pense le Logos à partir de la lecture : c'est, au contraire la lecture qu'il convient de penser à partir du Logos, comme étant une manière de glaner le sens, de le collecter et de mettre en réserve.

\section{Le Mandat (das Geschick) et le mandaté (das Geschickliche) : assigner (schicken)}

31 Parallèlement à cette première détermination du Logos, comme ce qui se donne à lire et qui est explicitée à partir de l'appariement de legen et de lesen et à partir du déroulé de la métaphore de la moisson à laquelle renvoient ces termes, Heidegger met en lumière une deuxième détermination qui lui est inspirée par d'autres jeux de langage qui gravitent autour de la thématique du destin (Geschick) qui allie, dans un même geste que Lacan traduit par Mandat, l'acte d'envoyer (schicken) et celui de recevoir (das Geschikliche). Le Logos est au principe d'une assignation, d'un appel, d'une adresse et d'un envoi ou, pour reprendre les termes du traducteur, d'une "grâce ", en vertu de laquelle il est le lot (Schicksal) échu et dévolu aux mortels ${ }^{15}$. La traduction de Préau reste timide : ce qui est destiné ne relève pas d'un ordre, mais d'une convenance et l'accueil qu'en fait le mortel le rend habile (geschikt), «bien disposé », lui confère le "chic ». Celle de Lacan suit le fil des signifiants du don et du Mandat et exprime la bonne disposition générée par le legein, en parlant de "cachet", terme qui, sans exclure le «chic», renvoie au monde de l'écriture : le Logos estampille l'homme, lui 
donne du caractère et lui confère ses caractères. Impossible d'échapper au legs qui oriente notre destin et à l'écriture qui porte la marque sigillaire du testament.

Cette détermination historique du Logos est inscrite dans le terme grec «sophon » que l'on traduit communément par "sage »: «il est sage de dire pareillement à ce sens : l'Un est toutes choses ». La question est de savoir ce que signifie ici « sage ». Heidegger propose une écoute événementielle du fragment d'Héraclite : on ne peut se contenter de traduire : «Il est sage de dire le même (homologein) », mais il faut traduire : « De dire le même, voilà qui témoigne du "sophon ", de la force du Mandat, qui oblige le mortel à se maintenir dans le champ de cette assignation. Ce n'est pas en termes de saisie intellectuelle qu'il convient d'interpréter le mot "sophon», mais en termes de comportement ou de maintien (Verhalten, Haltung). Mais, précise Heidegger en citant un autre fragment d'Héraclite (En to sophon mounon: l'Un seul est le Sage)), l'adjectif neutre «sophon », qualifie le logos humain, alors que le substantif « to sophon » qualifie le Logos lui-même : L'Un seul est le Sage ${ }^{16}$.

Lacan n'hésite pas à recourir au terme " mandaté ", pour caractériser le comportement du mortel et au terme "Mandat» pour signifier l'assignation que le Logos fait à l'homme : «Ainsi sophon veut dire cela qui peut se soutenir de ce qui lui a été assigné, y trouver son lot, s'y vouer, prendre la route pour cela. C'est par sa destination que ce maintien est commandé. Nous employons encore "idiotiquement", si nous voulons dire que quelqu'un est particulièrement habile en une chose, ces tournures : qu'il a la grâce pour cela, qu'il y met son cachet. C'est là plutôt que nous touchons à la signification propre de "sophon » que nous traduirons par " mandaté » ${ }^{17}$.

Autant de termes qui ont pour effet de faire, mais sans le dire, référence au signifiantmaitre : celui de loi et, en même temps, de mettre l'Un en abîme, de l'affecter de cette béance qui transmue l'impossible en Un-possible. Le Logos est le dévoilement d'un événement (Ereignis) dont on peut dire qu'il fait loi. Lacan, en parlant de Mandat, met ainsi en lumière l'instance "déontique » qui commande l'expérience "alêthique ". Heidegger tend à refouler le signifiant «loi » (lex) qui glisse sous le radical leg et vers lequel pointent les mots legs et lais, à la différence de Lacan qui ne peut se retenir de le convoquer en sourdine, sans jamais pour autant le nommer.

\section{Le signifiant élidé : la loi (lex)}

Il existe donc un Logos primordial qui, à bien l'entendre, s'énonce dans les registres de trois métaphoriques : celle de la présentation au-devant-de soi, celle de la moisson et celle de la lecture. Mais ces trois métaphoriques se recoupent dans un geste commun de rassemblement du sens qui, pour la première, fait le lit du repos de la présence, pour la deuxième, s'accomplit dans une mise à l'abri ou dans une mise en réserve de la moisson et, pour la troisième, se dépose dans des configurations signifiantes qui se donnent à lire. Mais cet événement advient de lui-même, sans qu'il n'ait besoin de personne pour le produire. C'est le Logos lui-même qui survient ainsi dans le silence : c'est le Logos qui met à reposer le sens dont il est l'actuation, selon l'expression de Lacan et qui, on le présume, préside au rassemblement ou à la récollection de ce qu'il met à reposer.

Ce que Heidegger nous donne à penser, c'est qu'il y a un « muthos » du Logos et que c'est précisément ce «muthos » qui reste à penser. Point besoin d'épiloguer longtemps sur cette collusion, pour comprendre la séduction que cette thèse a pu exercer sur Lacan. Ce n'est pas sans une certaine jubilation que ce dernier exprime ce rassemblement dans 
les métaphores du lit de la reposée où se lit le sens. Mais surtout, il sait rendre avec bonheur le mode de ce rassemblement en recourant au terme français de «legs » qui exprime, non sans une complicité euphonique avec le legein grec et le lesen allemand, le don ou la donation qui est l'essence de la présentation $d u$ Logos, au double sens du génitif objectif et subjectif. La traduction de Lacan n'est nullement fantaisiste, car le mot « legs » qui signifie testament découle du radical indo-européen leg d'où est issu le verbe grec legô entendu au sens de choisir et de cueillir et a donné naissance, comme on l'a noté, au mot legem, accusatif du substantif latin lex, la loi. Le traducteur qui convie, pour faire écho à ces termes, le mot lais, n'ignore pas que ce dernier a été le synonyme de loi en vieux français.

Lacan croise avec adresse les significations de ces deux axes lexicaux, en élidant intentionnellement celui de loi auquel ils renvoient pourtant l'un et l'autre. Le Logos nous est échu en héritage et il fait loi: le Logos se présente et il est lui-même sa propre présentation. Il laisse-être et fait-être (lassen) : tel est le propre du legs, mot qui, et c'est ce qui rend problématique sa référence à la racine leg, vient du latin relaxare, lâcher, laisser, transmettre. Le lais et le legs expriment les deux modalités de cette présentation dont la première est seulement suggérée : le Logos comme loi lie ce qu'il cueille et, par conséquent, le rassemble et, comme legs, il recueille ce qu'il rassemble ; il le fait être en le laissant être : il met à reposer : « Legein est mettre-à-reposer. Mettre-àreposer est le recueil en soi-même du fait de se laisser présenter ce qui est ensemble à être présent ${ }^{18}$.

\section{La question : le dire}

L'événement du Logos originel n'est pas pour autant élucidé par la magie des mots, car legein a aussi le sens prosaïque de dire et de parler. Une question surgit alors : "Jusqu'à quel point le sens propre de legein, mettre-à-reposer, en arrive-t-il à signifier ce qui se dit et se prononce? ». Heidegger répond en soulignant que ce passage s'effectue à la faveur d'un détour par l'écriture et la lecture. Ce qui n'est pas pour déplaire à Lacan : « Mettre en ce lit est donné à lire. La lecture qui nous est la plus connue, à savoir celle d'un écrit, reste, si prégnante qu'elle soit, n'être qu'un des modes du colliger, au sens de ramasser-en-présentant " ${ }^{19}$. Mais d'où provient cette écriture qui se donne à lire et à dire? La réponse tient à la présentation même du Logos qui met en lumière et met à l'abri "l'être de la présence de ce qui se présente dans le dévoilement " ${ }^{20}$, à savoir l'être de l'étant, car "...la révélation de ce qui est voilé dans le dévoilement est la présence même de ce qui est présent ${ }^{21}$. Ainsi, la Lettre est-elle la marque et la remarque de ce jeu de présence et d'absence, le trait du retrait de la présence. Le Logos se fait Lettre dans le jeu des re-traits et c'est à lire ces derniers que les mortels peuvent dire et parler. Tout se passe comme si une archi-écriture opérait la médiation entre le prologue et le dia-logue. La Lettre est la configuration symbolique du réel qui nous échappe : elle est la marque du refoulement.

Mais cette lecture est aussi un « ouï», une écoute silencieuse de ce qui passe et repasse dans le Logos, de ce qui se passe dans le Logos : le legs, jeu de passe et de passage... Un tel ouïr n'est pas affaire d'oreilles, mais d'une entente qui s'accorde au Logos qui nous est adressé et qui constitue notre-être-au monde : «Ce n'est pas que nous entendions parce que nous avons des oreilles. Nous avons des oreilles et pouvons être équipés d'oreilles parce que nous oyons. Les mortels oient le tonnerre du ciel, le bruissement de la forêt, le murmure de la fontaine, la touche vibrante de la harpe, le ronflement des 
moteurs, la rumeur de la ville, seulement et seulement pour autant qu'à tout ceci déjà ils appartiennent et n'appartiennent pas ${ }^{22}$. En conjuguant avec malice le verbe " ouïr ", quitte à faire crisser l'oreille, Lacan entend marquer, de façon radicale, la distinction entre les deux régimes de l'écoute: celui d'une écoute première que l'on qualifiera de signifiante, car elle fait advenir le sens et celui d'une écoute seconde que l'on qualifiera de symbolique, car elle est l'œuvre d'un « entendement » qui mobilise la compréhension.

\section{Homo-loger et homo-léguer : ó $\mu \circ \lambda \circ \varepsilon^{2}$ v (homologein)}

Homologein, mot qui signifie étymologiquement "dire le même", caractérise l'articulation du Logos primordial au parler humain. Il se réalise par le croisement du lire et du « ouïr » qui fonde l'entente avec « le lais où se lit ce qui s'élit » et avec le legs qui se livre à l'écoute des mortels. Il ne désigne ni l'énonciation de la personne qui lit et dit que le Logos vient à l'effectivité, ni l'opération d'un " ouïr » qui serait conforme au Logos. L'homologein exprime la correspondance que le mortel entretient par nature avec le Logos et l'attention, "grâce » à laquelle il s'y accorde. Comme tel, il nous renvoie à notre appartenance au Logos originel, qui nous habilite à écouter. Encore faut-il que nous acquiesçons à cette appartenance. Ce verbe cardinal ne vise pas, «bien entendu », à définir la posture d'Héraclite qui se ferait le porte-parole du Logos ni même celle de quiconque « saisirait » le sens du message, mais il caractérise le comportement ou le maintien qui doit être celui de nous tous, mortels, assignés par destin à l'écoute du Logos : kein blosses Erfassen, sondern ein Verhalten ${ }^{23}$. La traduction de Lacan nous enseigne que l'entente est commandée par l'Autre : «Ce n'est pas vers moi que vous devez tendre l'oreille (comme on fixe du regard), mais ce qui est d'ouïr pour le mortel doit s'orienter sur quelque chose d'Autre " ${ }^{24}$. En parlant de ce quelque chose d'Autre, là ou Préau parle de se tourner vers autre chose, Lacan nous renvoie, en passant, au lieu tiers qui commande tout discours et tout dialogue. Ce qui signifie que c'est du Logos que l'on écoute le Logos, que l'ouïr «se détermine à partir du Logos " ${ }^{25}$ ou, pour le dire plus radicalement, que «ce mode d'ouïr » est lui-même un «legein ». L'appel à l'accord est constitutif du Logos dont il accomplit la destination.

41 Mais une précision s'impose à ce stade : cette écoute est elle-même un legein particulier qui se réalise sur le mode d'un homologein. On dira alors que l'homologein est et n'est pas le legein : il est la manière dont le mortel se rapporte au legein, l'accueille comme un champ d'attente et d'entente. L'avènement du Logos fait événement dans l'homologein : le gîte se déplace en "ci-gît», le legs ne cesse d'être en avancement d'hoirie, pour s'accomplir comme lot échu. On ne saurait définir ce rapport en parlant de transposition (Préau) ni, même, à l'instar de Lacan, de transfert (Ubertragung) : la transposition ne modifie pas le contenu de l'audition et le transfert, s'il le modifie, se situe sur un parcours d'idéalisation qui va du régime de l'audition à celui de l'entente ${ }^{26}$. Or, c'est l'inverse qui se produit : c'est l'entente qui conditionne l'audition. Certes, il est plus juste d'évoquer le transfert plutôt que la transposition, mais cette double interprétation qui présuppose à tort qu'il y ait une spiritualisation de l'audition dans l'ouïr procède d'un mal-entendre (Préau) et d'un malentendu (Lacan) qui tiennent, l'un et l'autre, à une dénégation funeste de la priorité de l'ouïr, laquelle s'en trouve pourtant paradoxalement confirmée. C'est en réalisant «ce pur transport dans l'écoute » (sich verlegen), que l'homme lève une telle dénégation ${ }^{27}$. Lacan excelle à nous faire entendre que l'homo-logein est le dévoilement du legein comme legs et que ce legs 
laisse gésir, en l'homme qui s'y transporte, le legein comme lui étant semblable (homon). Là où Préau parle de conformité du legein mortel au Logos, Lacan, fidèle à la métaphore du legs, parle d'une " événementialité » qui se réalise sur le mode d'un lot : «Quand Le legein mortel trouve son lot dans le Logos, il arrive de ó $\mu \circ \lambda$ est ainsi au mieux traduit: "Wenn das sterbliche legein sich in den Logos schickt, geschieht homologein ${ }^{29}$.

C'est à la lumière de cette transition/transaction du Logos, que Heidegger interprète, en les confrontant, les deux mots énigmatiques d'Héraclite que sont sophon (sage) et monon/monon (seul). L' homologein est l'expérience du «sophon», mot qui signifie littéralement ce qui est sage, mais qui, dans ce fragment d'Héraclite, qualifie le maintien, la tenue du mortel qui, mandaté à la garde du legein, se porte garant du lais, devenant ainsi, comme le note Lacan, un re-lais. La traduction de Lacan suit le fil des métaphores : le relais laisse être le lais et le fait être en le faisant pareil au même. Celle de Préau, plus prosaïque, voit l'homologein réalisé par « celui qui se tient dans le séjour (Aufenthalt) des mortels ${ }^{30}$. Elle contraste avec l'interprétation de Lacan, qui inverse la perspective : dès que se trouve réalisé l'accord de l'homologein, alors il y a du "sophon ", car le mandaté ou plutôt « du mandaté vient à l'événement, pour autant que l'Un est Toutes choses ${ }^{31}$ : Geschickliche ereignet sich, insofern Eins alles ${ }^{32}$.

43 Ne croyons pas que cette posture s'impose par le seul décret de la volonté du sujet : elle lui est dictée par le Logos lui-même. Ainsi, le mortel est-il assigné au "sophon » (geschicklich), mandaté par le Logos qui en fait son légataire universel, son unique héritier, son com-manditaire. Il est depuis toujours mandaté et c'est l'oubli de cette condition qui lui fait rater son rendez-vous avec le Logos : « Quand le fait d'ouï proprement en tant que homologein est, alors vient à l'événement ce qui tient du mandaté, alors le legein mortel trouve son lot dans le Logos. Alors il colle au lais où se lit ce qui l'élit. Alors le legein se commande par la destination qui repose dans la répartition qui se produit de distribuant à l'origine, c'est-à-dire dans ce que le lais où se lit ce qui s'élit a commandé ${ }^{33}$. Lacan enchaîne les allitérations, pour chanter le privilège du mortel qui a la grâce de l'homologein .

L'homologein exprime l'accord de l'homme avec le Logos auquel il se doit d'appartenir (gehören) et d'obéir (gehorschen). Il a son principe dans l'écoute et son accomplissement dans le dire (sagen). Il suppose donc que celui qui parle se mette en phase avec le Logos, ce qui ne saurait se faire sans une décrispation de sa subjectivité. Mais il convient de bien définir quel type de rapport (Austrag) il ouvre. Point n'est question de l'interpréter comme un rapport d'entente qui lierait les membres d'un groupe, décidés à parler d'une voix unanime. Certes, une telle lecture politique pourrait être légitimée par l'ambiguïté des termes employés, comme entente, appartenance, élection. Mais ni le texte de Heidegger ni la traduction de Lacan n'avalisent cette interprétation. L'ouïr est le chemin qui mène au dire originel du Logos et ne recouvre ni le phénomène d'une entente collective ni le phénomène de l'écoute des mots proférés. Heidegger revient, en effet, à plusieurs reprises, sur la corrélation inédite existant entre la mort et le Logos : seuls, les mortels sont mandatés à ouïr et à dire.

Mais comment penser une telle corrélation? Sans doute en disant que le Logos n'a ni archê ni telos, qu'il survient comme étant l'événement d'une présence qui surgit de l'absence pour s'y replier, qu'il se décline indéfiniment dans le langage sur le mode de glissements de sens ou de différences signifiantes, qu'il échoue à s'accomplir dans un mot de la fin qui ressusciterait le réel perdu, autour duquel il mène la danse. Défiant 
tout ordre ontologique, il est l'être-à-la mort dont se nourrit la vie. S'il n'était cette énigme de l'Un/impossible qui rend Tout possible, il serait le Tout/Un mortifère qui asphyxierait le devenir vivant de l'être-à-la mort. Ouïr le Logos, c'est entendre la pulsation de la mort qui bat le rythme de cette incessante reprise des possibles dont s'enchante la vie.

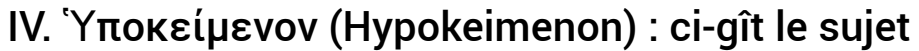

Voici que ce double passage du lire à l'ouïr nous met en correspondance avec le Logos et « renseigne » le dire : le sens ne tient ni à la signification que nous livre la lecture ni à l'expression vocale de la voix, mais à la survenue du Logos qui, en se dévoilant, fait le lit de la présence dans l'hypokeimenon. Rappelons que ce terme aristotélicien ne désigne ni la substance, au sens classique du terme, puisqu'il ne caractérise aucune réalité permanente, ni le sujet humain auquel Descartes l'a identifié, mais le rapport d'ouverture que le Logos entretient avec l'homme mortel, rapport qui donne lieu au lire et au dire. Or, si c'est dans ce rapport que se déroulent le choix et le rassemblement du sens, on peut dire que l'hypokeimenon qui sertit «le domaine marqué de l'empreinte originelle qui est l'essence du langage ${ }^{34}$ est le lieu même de l'être-là, du Dasein : «Dans ce legein de l'hypokeimenon, le legein du choix et de la collation demeure serti ${ }^{35}$ : In dieses legein des hypokeimenon bleibt das legein, als lesen, sammeln eingelegt ${ }^{36}$. L'hypokeimenon est le legs de la présence sous forme de dépôt et de suppôt, le ci-git du gite de la présence. En indiquant que le terme "hypokeimenon " garde la marque de la forme moyenne du verbe grec "keisthai » (allemand : für-sich-Vorliegen), Heidegger, sans pour autant y voir la désignation du sujet, en décèle les conditions et les contours, soulignant que le Logos est mis à l'abri dans la parole humaine et que c'est l'homme qui est le porte-parole du Logos (keisthai: «le fait d'être-étendu-pour-soi-devant », selon Préau ${ }^{37}$ et, selon Lacan, « le pour-soi de la présentation de ce qui est ainsi relégué du hypokeimenon $"{ }^{38}$. Ce terme-clef du vocabulaire philosophique caractérise finalement le sujet supposé du langage dont la présence, loin d'être thétique, reste toujours hypothétique.

Se dessine alors à l'horizon une formulation nouvelle de la question posée. Il ne s'agit pas de savoir comment le «mettre-à-reposer » se décline en dire et en parler, mais de savoir si le dire et le parler font l'expérience de ce "mettre-à-reposer », ou encore si la présence advient comme hypokeimenon, c'est-à-dire comme une configuration de traces qui délimite le ci-gît du Dasein mortel. Ainsi sommes-nous invités à prendre la mesure d'un événement de pensée qui nous appelle à un retournement (Kehre) de nos manières de penser : il n'y a point à passer du «mettre-à-reposer » au dire et au parler, car le dire et le parler ne se réduisent pas à des phénomènes de vocalisation ou de signification, mais sont eux-mêmes des modalités du " mettre-à-reposer ». Il nous faut nous-mêmes nous transporter dans l'expérience du « mettre-à-reposer ", afin de dire et de parler. En parlant, ne posons-nous pas nous-mêmes le sens et, dès que nous le posons à nouveau, ne le reposons-nous pas au double sens de ce terme? Le silence ne serait-il pas le langage de cette mise au repos? Écoutons la traduction de Lacan: « Ce que disent et prononcent les mortels advient dès l'aube à l'événement comme legein, comme fait de mettre-à-reposer... Le legein se laisse ainsi surmonter en tant que fait de mettre-à-reposer par cet aspect en lui prédominant (Das legein lässt sich als das Legen von dieser Art überwaltigen). La pensée humaine ne s'est jamais étonnée de cet événement et n'a pas perçu un mystère où quelque chose d'essentiellement destiné par l'Être à 
l'homme se dissimule, pour se réserver peut-être pour cet instant fatal où la semonce qui ébranle l'homme ne s'étend pas seulement à sa place et à sa condition, mais où elle porte l'essence de l'homme à la vacillation qui la fait bouger " ${ }^{39}$. Si nous comparons les deux traductions, nous constatons que celle de Lacan qui rend le verbe legen par "mettre-à-reposer " et le verbe "überwaltigen " par "surmonter " s'inspire d'une dialectique où repos rime avec répit et surmonter avec résister et dépasser, dialectique dont s'écarte Préau, en traduisant directement legen par « étendre » et überwaltigen par «dominer».

Le mortel qui parle n'est pas pour Heidegger le sujet au sens classique du terme. Reste que la traduction de Lacan laisse entendre, sur le mode de renvois et d'allusions, que le legs et le lais que Heidegger assimile à l'hypokeimenon aristotélicien, terme qui désigne traditionnellement le sujet grammatical fâcheusement interprété comme substance, pourraient bien faire le lit d'une conception inédite du sujet. Par l'accord qu'il noue avec le Logos, le mortel devient un porteur (Träger) du sens, en vertu du transfert (ÜberTrag) réalisé dans l'homologein. Si le Logos laisse (lassen) advenir le sens, il le fait aussi (lassen) advenir dans un accord qui épouse la forme contractuelle du Mandat. Lacan interprète l'homologein comme étant à la fois le principe et l'effet de la constitution de la subjectivité. Or, le profil d'un tel sujet, qui est lui-même emporté par l'énigme du Logos, est celui du mortel qui correspond à l'appel déontique du Logos surgi du creux de sa manifestation alêthique. Mais cette correspondance n'est ni occasionnelle ni habituelle : elle se présente comme une tenue (hexis) insistante mobilisant la volonté.

\section{L'un/Toutes choses : "Ev חávta (en/panta)}

49 Le Logos est l'Un qui est toutes choses: «... du mandaté vient à l'événement, pour autant que l'Un est toutes choses». Que signifie cet énoncé paradoxal? Heidegger écarte une interprétation qui banaliserait la parole héraclitéenne en s'appuyant sur un verbe supposé les unir. Selon une première "lecture ", il s'agit du verbe "savoir " (eidenai) et, selon une autre, du verbe "être» (einai). On dira alors, au gré du verbe convoqué, qu'il est sage de savoir que tout apparaisse comme l'Un ou encore qu'il est sage que tout soit l'Un. Mais cette double lecture ne met pas suffisamment en perspective l'événement de la survenue de l'Un comme Tout ou du Tout comme Un. Heidegger écarte ces deux " versions » dont la « correction » syntaxique fait écran aussi bien à l'écoute de l'homologein et à l'accueil du sophon qu'à celle de l'interprétation qui unit l'Un et le Tout. Il suffit de dire l'Un-Tout. La parataxe fait écho à l'événement d'une articulation originelle : « Il y a le Logos. Celui-ci a quelque chose à annoncer. Il y a alors aussi ce qu'il annonce, à savoir que l'Un est toutes choses $"{ }^{40}$. Elle permet de focaliser notre attention sur l'énigme à déchiffrer, sur l'effet d'annonce d'une parole qui ne tient pas à ce qu'elle énonce, mais bien à ce qu'elle dénonce de la nature du Logos.

Écartons les malentendus. L'Un peut être interprété comme étant l'Autre qui sous-tend le dire et le parler et, de son côté, le Tout ne désigne ni une totalité fermée ni le milieu de la dissémination de toutes choses. Les deux termes sont réversibles : ils se situent dans un rapport où chacun assume la fonction de l'autre. L'Un unit et répartit, le Tout distribue toutes choses et les sauvegarde dans leur unité : «En est l'Uniquement-Un en tant que ce qui unit. Il unit en même temps qu'il répartit... Ce fait ici d'unir ce qui dans ce lit se lit, répartit en soi ce qui unit jusqu'au point qu'il est un-ci, et en tant qu'il est cet un-ci, aussi l'Unique $"{ }^{41}$. L'Un est ainsi l'Unique : le singulier-pluriel qui unit et 
distribue toutes choses. Il pointe la différence qui articule toutes choses. Si le Logos rassemble et s'étale, si le Logos legei, c'est le En, l'Unique et le Tout, qui en constitue les deux modalités fondamentales: L'En/Panta nous fait entrevoir «la manière dont le Logos se déploie dans son legein ", traduit Préau, alors que Lacan, plus fidèle au texte, précise avec bonheur qu'il « contient le cillement d'ouverture (Winck) dont le Logos est essentiellement dans son legein » ${ }^{42}$, le « clin d'œil » invitant à l'écoute.

51 Si l'Un est l'Unique, c'est parce qu'il relève du Logos : il est, de ce point de vue, soustrait à toute interprétation ontologique. C'est le langage qui fonde l'ontologie. Ce qui signifie que l'être n'est pas du ressort d'un ordre réel, sur lequel on peut discourir, mais qu'il est un effet du symbolique. L'homme est un "parlêtre», parce qu'il est d'abord un "désêtre ». Le Logos n'est pas un rapport unissant deux termes : le langage et l'homme. Il est avant tout une ouverture antéprédicative. Heidegger, et, à sa suite, les deux traducteurs distinguent avec force le dépliement ou le déploiement du legein du rapport qui se réalise dans l'homologein. Entre le legein et l'homologein, il y a un écart : celui de l'élection, souligne Lacan, celui de l'événement qui ouvre la présence, note Heidegger. Ce qui revient à dire que le Logos comme tel est un non-rapport qui rend possible toute mise en rapport. Il est l'Unique, mais il se présente sous la forme paradoxale de l'Un et du Tout. C'est dire qu'il est, comme Un, avec son corrélat, le Tout, un pôle de la relation, mais, comme Unique, le tiers instituant de la relation.

52 L'Un dont il s'agit n'est pas l'Un conçu comme le genre commun (koinon) qui rallie les éléments du Tout et les uniformise. Il n'est pas normatif, mais soustractif : c'est en disparaissant dans le Tout qu'il s'en extrait, laissant le trait unifiant de sa propre soustraction. Il fait effraction dans le langage comme le trait d'esprit qui rassemble les mots, en les renvoyant à un sens inédit. C'est, en effet, dans un jeu d'écriture que Heidegger pense le rapport de l'Un au Tout. Préau traduit littéralement le texte allemand: Dieses Zeichnen bliebe eher ein freiwagendes Vorzeichnen als ein selbst sicheres Nachzeichnen ${ }^{43}$, en parlant de dessin (Zeichnen), car l'Un du Logos se profile dans toutes choses, s'y anticipe, s'y pré-dessine (Vor-zeichnen) : « Dessiner ainsi, ce serait plutôt prédestiner librement, à ses risques et périls, que suivre un modèle en toute sécurité ». Lacan, de son côté, traduit en insistant sur l'esquisse (Vor-zeichnen) et le dépôt des traits qui président au profil du tracé : «Dût ce tracé demeurer une esquisse d'une libre audace plutôt que viser à la certitude d'une étude d'après le modèle ${ }^{44}$. Mais qu'il s'agisse du trait du dessin ou de celui de l'écriture, c'est sous la forme de traces que le legein se déploie comme Un et Tout. Car l'Un qui est l'Unique (das Einzig-Eine), qui est le Trait d'union, le Trait unique, se présente comme un simple signe (Préau) ou mieux, comme le traduit avec justesse Lacan, fait signe sur le mode d'un cillement (En-Panta gibt den einfachen Winck in das, was der Logos ist ${ }^{45}$. Il cille et vacille en toutes choses. Tout se passe comme si les traces de l'Un se trouvaient effacées par les tracés de toutes choses, de sorte que c'est après coup que l'on peut saisir, en recomposant les configurations diverses du Tout, l'effectivité et l'efficacité symboliques de l'Un.

53 La pensée n'est-elle pas menacée de s'égarer en posant le Logos à partir du logos mortel, comme s'il était le fruit d'un transfert d'un trait (Zug) caractéristique de ce dernier sur le mode d'un «surhaussement » ou d'une « sublimation ", termes qui traduisent le mot allemand «Übersteigerung " ${ }^{46}$ ? On se tromperait tout autant à évoquer, à l'inverse, une relation de mimêsis, qui ferait du logos mortel une imitation (Nachbildung) du Logos comme tel. Autant de voies où la pensée grecque s'est fourvoyée à ses débuts et qui ont, comme le traduit Lacan, barré (verlegt) et brouillé (verrätselt) ce que le Logos a 
d'essentiellement énigmatique (rätselhaft). Il nous faut délaisser ces explications qui paralysent le questionnement. Il en est ainsi : l'être des mortels est, selon la version de Lacan, "dévolu» ou, selon celle de Préau, «transproprié » à l'homologein (dem homologein vereignet) : il est assigné à cette tâche, homologué pour l'accomplir. Le Logos exprime la manière dont l'Être de l'étant se donne à dire en même temps comme multiple sur le mode "distributif» et comme Un sur le mode "rétributif», pour reprendre les mots de Lacan. Notre époque a oublié que l'homologein doit se plier à la Mesure du Logos, parce qu'il " faut » au Logos l'homologein (Weil der Logos das homologein braucht). Il le faut: Heidegger nous a indiqué que le terme "brauchen » que l'on traduit communément par « avoir besoin » signifie étymologiquement « jouïr » et la jouissance dont il s'agit est de l'ordre de l'ouïr ${ }^{47}$. L'homologein est le jouir de l'ouï : jouissance de l'accord au Logos qui fait ouir. C'est cette dimension essentielle que rature la modernité en substituant au jouïr/ouïr éprouvés dans l'homologein l'exigence du faire (Tun) de la technique qui entraîne la démesure de l'oubli de la venue à la présence de l'Être comme sens du Logos. Mais Lacan ne s'engage pas dans cette voie.

L'Un se démultiplie dans des uns pluriels qui, par ricochets, dessinent les cercles concentriques du Tout. Le sens, qui est à la fois Un et Tout, est un effet du symbolique. Dès que l'on perd de vue le littoral de cette interprétation, on prend pour réel ce qui relève du symbolique. Mais, s'il n'est levé, ce malentendu laisse la porte ouverte à toutes les orthodoxies, à tous les dogmatismes, à tous les totalitarismes, aux névroses et aux psychoses individuelles ou collectives. Contrairement à ce que l'on imagine, ce n'est pas l'Un qui fait le Tout, mais c'est le Tout qui convie l'Un à s'y faire. Mais bien sûr, ce Tout n'est ni une totalité, ni une totalisation en devenir. L'Un, c'est ce qui unifie le Tout en le convertissant, par sa soustraction même, en "pas-tout ». Il est l'Autre, l'« en-plus » qui lie toutes choses.

Heidegger renvoie le lecteur engagé sur le chemin de cette énigme à l'interprétation qu'il a entreprise dans L'Être et le temps (Sein und Zeit,\&7B): L'Un, c'est le Logos qui " porte ce qui apparaît, ce qui se produit en se présentant, de ce qu'il est en lui-même à ce qui paraît, à ce qui se montre comme tiré au clair » ${ }^{48}$. Autrement dit, il unit ce qui se distribue dans la présentation « rassemblante » qu'il en fait. Dire, c'est recueillir cette présentation, c'est présenter ce recueillement et, par conséquent, faire venir l'Être à la présence. Le Logos promeut la présence et, en s'effaçant, y reconduit. Mais une telle présence est bien celle de l'Être-Un de l'étant, c'est-à-dire celle de l'Être de toutes choses qu'il porte à la lumière : « Le En Panta laisse se présenter ensemble dans l'Être d'une présence, ce qui est essentiellement divergent l'un de l'autre et s'oppose ainsi l'un à l'autre par une absence contrariée, comme le jour et la nuit, l'hiver et l'été, la veille et le sommeil, Dionysos et Hadès ${ }^{49}$. On le pressent, la différence ontologique que Heidegger situe entre l'Être et l'étant se trouve ici pensée dans l'espace du Logos mesuré par la différence entre l'Un et le Tout. C'est bien cette différence qui est « amenée à la parole », mais aussi effacée par la parole qui l'énonce. L'oubli de l'Être est l'oubli de l'Être qui s'énonce dans le Logos.

Le Logos ne réalise pas une banale connexion ou liaison de toutes choses. Il les lie en les unissant dans la présence : s'il lie, c'est parce qu'il est le lais, le sens qui repose dans le lit où se lit ce qui s'élit. Cette dérive de jeux de mots, cultivée par le traducteur, nous rappelle, d'abord, que c'est le Logos comme tel qui se donne en tribut aux mortels : il fait être ce qu'il laisse être, il se délègue/dé-legs, il commande le Tout et l'Un: il se distribue dans le tout et se rétribue dans l'Un. Ce n'est pas le mortel qui a l'initiative du 
Logos, mais c'est le Logos lui-même qui éveille le mortel à ce Logos qui parle en lui la même langue. Mais ce qui s'élit du Logos n'est pas pour autant le seul privilège du Logos primordial : le mortel lui-même, par son homologein, participe de cette élection, se l'attribue, puisque c'est par lui que le Logos parvient à se dire, mais aussi participe de cette élection, puisque il a la possibilité de s'accorder au Logos et celle de s'en désaccorder. Il a donc la capacité de recueillir, par son dire, ce qui se recueille, de lire et d'ouïr ce qui s'élit.

Le Logos fait surgir et perdurer la présence dans le présent dévoilé de toutes choses. Il en assure la sauvegarde, mais en lègue le pouvoir de la reconduire au logos du mortel: «Par lui est sauvegardé tout ce qui est présent dans l'être de sa présence, à partir de quoi il peut être rentré et ressorti tout exprès comme ce qui est présent à un instant quelconque par le truchement du legein mortel. Le Logos promeut l'être de la présence, et reposant aussi ce qui est présent dans l'être de la présence, il l'y reconduit. S'apprésenter veut dire pourtant: une fois surgi durer dans le dévoilement $"{ }^{50}$. Ce faisant, ce que réalise le Logos, c'est le dévoilement de ce qui est présent, de toutes choses, dans l'être de sa présence. Mais on ne saurait parler de dévoilement sans faire référence à un voilement qui le précède ou l'excède : les choses dévoilées (alêthéa) sont telles, parce qu'elles sont arrachées au voilement: "Tout ce qui est de révéler délivre ce qui est présent du voilement. Le fait de révéler a besoin du voilement. L'A-Lêtheia repose dans le Léthé, puise en lui, produit ce qui par son travers est relégué. Le Logos est en soi à la fois une révélation et un recel. Il est A-Lêtheia $»^{51}$.

\section{Zeus : figure symbolique de l'Un}

Cette révélation qui déchire la nuit du voilement a pour symbole l'éclair divin qui assigne chaque chose à l'ordre de la présence. Le Logos est le mandant qui commande Tout. C'est lui qui produit la fulguration de la présence. Héraclite ne dit-il pas : «Toutefois, l'ensemble de tout..., c'est l'éclair qui le gouverne " ${ }^{52}$ ? L'éclair, c'est le Logos qui est la lumière qui fait advenir le monde dans son "omnitude», terme que Lacan invente sans doute, pour faire entendre, mais sans le relever, qu'il s'agit bien de l'«(h)omnitude », marque plénière de la finitude. L'éclair désigne Zeus lui-même. Le Logos qui se déploie comme En-Panta serait-il le Dieu suprême? La réponse à la question n'est pas aussi simple, dès lors que l'on se réfère à cet autre fragment d'Héraclite (B 32) : «L'Un, le Seul qui soit le Sage, ne veut pas et veut pourtant qu'on le dénomme du nom de Zeus ». Si l'on fait l'exégèse du verbe ethelein, vouloir, on découvre que le sens n'est pas celui d'une pure exigence, mais, comme le souligne en note J. Lacan, celui d'un " consentir à ». Or, selon l'interprétation qui a été faite du Logos comme En, on comprend que Héraclite admette que le Logos «ne consente pas à être recueilli (legesthai est le verbe legein au sens passif) sous le nom de Zeus " ${ }^{53}$. Sinon le legein perdrait sa signification originelle, celle de « léguer » et d'élire, pour désigner le fait de parler, de dire et de nommer et revêtirait la figure de l'Un absolu. Et pourtant, poursuit Héraclite, l'Un est aussi disposé à consentir à ce qu'on le dénomme du nom de Zeus. La question se précise, dès lors que l'on remarque, comme le traduit Lacan, que ce double énoncé exprime une dénégation (Verneinung) plutôt qu'une négation (Préau), car, selon Heidegger, le «ne pas» qui accompagne le verbe "consentir» (en grec: mê et non ouk) exprime une négation qui n'est pas absolue, mais relative et, du coup, devient problématique, car on peut se demander si une telle dénégation a bien «sa raison dans la chose $»^{54}$. 

qui est affaire de projection et l'apparaître qui est affaire de nomination, mais aussi, par voie de conséquence, à la capacité que recèle la parole humaine de nommer les choses à l'appel du Logos. C'est, en effet, le langage humain qui arrache les choses au paraître et, qui, en les nommant, les fait apparaître. Mais ce pouvoir de nommer, il le détient du Mandat du Logos lui-même. On le comprend, la dénégation n'est point ici une figure de rhétorique, car elle exprime le pouvoir inhérent au Logos de dire et de se dédire, c'està-dire de voiler, en le dévoilant, ce qu'il indique: d'apparaître, à l'appel de la nomination, sous les traits de Zeus et de disparaître, en se retranchant dans le secret de son être. Elle est au principe du langage qui, sans elle, ne peut faire signe. Grâce à elle, la parole fait ciller les choses. Grâce à elle, le Logos nous fait signe, en épousant les traits du Dieu suprême.

60

eidegger voit la raison de cette dénégation dans la chose elle-même, c'est-à-dire dans l'articulation de l'Un/Tout. Il y a, bien sûr, d'un côté, le En, l'Un du Logos, lais qui laisse être dans sa présence tout être présent, mais qui n'est en aucune manière un étant présent entre autres et, d'un autre côté, l'ensemble des choses présentes, des étants, ta panta, qui ne sont dites présentes que par la «grâce » (Geschick) de l'Un. Or, Zeus est une exception : d'une part, il ne saurait être l'Un qui est l'Unique absolu, sauf à le détruire comme tel et, d'autre part, il ne saurait être réduit à n'être qu'un être présent entre autres, car il est « le plus haut de ce qui est présent ${ }^{55}$. Zeus n'est pas l'Unique et n'est pas un parmi d'autres, mais il est unique en son genre. Selon Heidegger qui commente le fragment VIII de Parménide, il préside à la partition (Moira) de l'être de la présence. Il est, selon la traduction de Préau, « le destin du Tout » ou, selon la formulation de Lacan, qui joue sur le signifiant " la grâce de l'omnitude ». Entendu comme Un, au sens d'Unique, le Logos se manifeste sans consentir à une quelconque dénomination qui le ravalerait à la position d'un étant entre autres, fût-il l'Étant suprême. Mais entendu comme étant le Tout, ta panta, le Logos se manifeste, " alors et alors seulement », précise Heidegger, comme étant l'« entièreté » ou l'« omnitude » de ce qui est présent sous le gouvernail de Zeus. Ainsi dénommé, il se présente, note le traducteur comme «l'Entier de l'entièreté », expression qui consonne heureusement avec la définition latine du Dieu de l'onto-théologie : « Ens entium », l'Étant le plus haut des étants.

Il y a donc deux manifestations qui se croisent : la manifestation an-archique du Logos, conçu comme l'En, le seul « tô Sophon », qui exclut toute référence à Zeus, car il est « le mandat en tant qu'il est la grâce elle-même " ${ }^{56}$ et la manifestation hiér-archique (de hieros, sacré et archein, commander) du Logos comme Tout, qui, en en appelant à Zeus, est habilité à conférer à l'ensemble des êtres présents l'unité fulgurante qui accomplit " les mandements de la grâce (die Schickungen des Geschickes) " ${ }^{57}$. Zeus est, en quelque sorte, le trait d'union entre ces deux versants du Logos: celui de l'Unique absolu et celui de l'Un relatif au Tout qu'il rassemble. Il représente, pour reprendre l'expression de Lacan interprétant Freud, "ce trait (l)unaire ", à tout le moins fulgurant, que fixe le nom de Zeus érigé en premier relais du lais, en délégué du legs. On dira que le nom de Zeus s'inscrit dans le schème d'une unité et d'un rassemblement symboliques qui sert de médiation entre l'Unité primordiale du Logos et l'Unité cardinale de la totalité des êtres. Telle est le milieu d'une possible expérience religieuse qui, selon la double étymologie du terme, fournit un relais au Logos (religare) et en fournit une lecture (religere). Zeus est le nom de l'onto-théologie antique, qui est toujours une onto-typologie, car elle projette le Logos dans une panoplie de figures et 
une thé-odyssée, car elle obéit à la logique alternée de l'exil hors du chez-soi et du retour au chez-soi.

Levons, au passage, le malentendu qui pèse sur l'interprétation de l'onto-théologie par Heidegger : l'onto-théologie est, à côté de la subjectivité et de la rationalité technique, une modalité du dévoilement historial de l'Être, non une modalité entre autres, mais la modalité la plus éminente, puisqu'elle focalise, entre l'Être et l'ensemble des étants, l'ordre d'une transcendance garante de l'unité. Il faut aussitôt ajouter que cette transcendance n'est pas fondée sur un rapport de causalité, comme tel est le cas du discours théologique médiéval, mais sur un rapport de nomination. La question est bien de savoir ce que nomme le mot Dieu ${ }^{58}$. Le texte «Logos » en définit les conditions de possibilité et de validité.

Nous pouvons mesurer le chemin parcouru par la pensée en comparant la traduction courante du fragment: "Il est sage que ceux qui ont écouté, non moi, mais le discours conviennent pareillement que l'Un est Toute chose » ou encore l'une des traductions reçues que reprend Heidegger : "Si ce que vous avez entendu n'est pas de moi, mais du sens, il est sage aussi de dire pareillement à ce sens : l'Un est toutes choses ", à la transposition finale qui résulte du déchiffrement du palimpseste, où la parole dite en grec se lit, furtivement, en latin, et, avec insistance, en allemand, avant d'être traduite et interprétée en français par Lacan. La traduction française met l'accent sur l'homologein qui réalise l'entente du lire et du ouïr, puis sur l'homologein en tant qu'il est une modalité du legein lui-même, enfin souligne avec force, à partir des jeux de langage de type juridique (le mandat, le mandaté, le tribut) et religieux (la grâce), ce qui du Logos fait événement: Toutes choses en l'Un. Rappelons le texte final de cette perlaboration langagière qui se passe désormais de commentaire :

Non de moi, mais du lais où se lit ce qui s'élit, en entente : cela même le mettre à sa place : et que ce qui est mandaté soit (le lais où se lit ce qui s'élit); l'Un en tant qu'unissant toutes choses ${ }^{59}$.

\section{Lacan interprète de Heidegger}

Le lecteur averti a sans doute entendu, au fil de la traduction, résonner les thèses fondamentales de Lacan: le Logos, assimilé à la vérité, se dévoilant dans la parole ; l'accès à l'inconscient s'effectuant par l'écoute du dire; la présence de toute chose surgissant de l'effacement du signifiant; le parler humain se réalisant sur fond d'un oubli ou d'un refoulement du Logos originel; l'impossibilité d'oublier l'oubli ou de dissimuler la dissimulation; enfin, la conception de l'homme qui, trouvant sa mesure en lui-même, dans le Logos qui l'habite et qu'il habite de ce fait, existe dans la différence qui, ouverte par ce dernier (legein), fonde la référence à soi (homologein) et à autrui. On a sans doute perçu, tout au long du commentaire, que la réception de la philosophie de Heidegger par Lacan se fonde sur l'idée que le dévoilement de l'Être et celui du désir sont en congruence, puisqu'ils se réalisent, tous les deux, par la médiation du Logos.

\section{I. À la croisée des chemins}

Mais la distinction, voire l'opposition existant entre ces deux mondes de pensée se trouve, sinon gommée, du moins relativisée par la traduction. Car la pensée de l'Être et 
l'écoute de l'inconscient mobilisent un "acheminement vers la parole» qui conditionne, à titre de présupposé épistémologique et méthodologique, aussi bien l'herméneutique phénoménologique que l'acte d'interprétation analytique. Heidegger, comme Lacan, récuse toute ontologie qui ne s'inscrirait dans cette dynamique du Logos. Déjà, pour Husserl, le phénomène offert à la conscience n'est jamais perçu à la même place ni sous le même aspect: comme le signifiant, l'être du phénomène est néant, abîme circonscrit par les visées intentionnelles. Il ne peut être saisi comme tel, en totalité, mais dans une succession d'apparitions qui rendent vaine toute recomposition ultérieure de la chose. Ainsi se réduit-il lui-même à la pluralité des aspects qui s'emploient indéfiniment, sans jamais y parvenir, à le circonscrire : ce qui le constitue, ce n'est pas une chose en soi, mais le vide. Tout en assumant l'héritage de Husserl, Heidegger prend au mot la phénoménologie et fonde l'herméneutique qui l'explicite sur une ontologie de la parole : l'être du phénomène comme tel est le Logos, qui n'est jamais adéquat aux paroles dont il est le principe.

On perçoit dès lors plus précisément les analogies existant entre l'herméneutique phénoménologique et l'acte d'interprétation analytique : l'Être de Heidegger, comme l'inconscient de Lacan, sont deux manières d'inverser le rapport métaphysique de l'être et du Logos : il n'y a pas d'abord de l'être duquel et sur lequel on parle, mais le Logos et ce qui du Logos fait être. Autant dire que l'être est une invention, c'est-à-dire le produit d'une révélation et d'une construction langagière. C'est ce renversement de perspectives que partagent Lacan et Heidegger lisant Héraclite. Si le Logos est cet événement qui se donne à lire et à dire, on peut présumer qu'il se présente au philosophe comme la déchirure d'une présence en sursis et, pour le psychanalyste, comme une "béance causale » ou, pour employer un autre terme de Lacan, «comme une béance pré-ontologique ». Il est, à leurs yeux, le principe du sens en devenir, de la dialectique inépuisable d'un non-être actif qui n'est ni de l'être, ni du non-être, mais qui est, du " non-né », c'est-à-dire du non-advenu ou encore du « non-réalisé », c'est-àdire du réel. C'est le non-être, qui est la source d'un dire en mouvement: il nous enseigne que c'est l'abîme qui parle, dès lors que le mortel a décidé de l'ouïr : «L'inconscient, d'abord, se manifeste à nous comme quelque chose qui se tient en attente dans l'aire, dirai-je, du non né ${ }^{60}$.

\section{Logos et la structure : l'inconscient}

Le Logos surgit comme une faille dans l'être : comme une béance dont il faut que le sujet prenne la charge (Träger), sans craindre d'affronter l'absence. Mais, par cette décision, il décède, car il consent, par l'homologein, à assumer l'effet aliénant de son implication dans le Logos, c'est-à- dire à renoncer à l'impossibilité de se fonder. On peut dès lors expliciter ce que Lacan pointe dans la traduction : c'est l'impossibilité, l'à-mort du sujet, sa castration ou, pour reprendre le terme aristotélicien qu'il convoque, la disparition (aphanisis) qui l'éveille à la vie. C'est l'évanouissement du sujet, aliéné par son implication dans le Logos, qui commande son surgissement. Cette vie qui se fait et défait au fil de l'à-mort, au fil de l'assomption de cette disparition, c'est bien la vie.

Il y aurait alors deux formes d'aliénation: celle, mortifère, qui ferme la porte à l'àmort, à tout retour du signifiant, et convoque, par cette forclusion, la mort réelle sur le champ/camp et celle qui, en assumant l'à-mort, ouvre l'espace du devenir de la vie. La traduction de Lacan, qui s'inscrit dans le mi-dire, tire le texte de Heidegger vers la 
seconde interprétation. Mais on peut oser une autre interprétation : celle qui consiste à reconnaître que cette alternative n'est jamais clairement posée comme telle et se présente, chez les individus et dans les groupements humains, comme une dangereuse alternance. Comme le souligne Heidegger, c'est la tenue (Haltung) du sujet dans le Logos, donc dans l'absence, qui tient lieu de fondement.

Le Logos rassemble ce qu'il disperse. Il est l'Un/inconscient, qui, «structuré comme un langage », est effet du signifiant-un et cause des signifiants-tout, au travers desquels il se dévoile. On peut comprendre que les métaphores du rassemblement, du recueil et de la moisson aient trouvé chez Lacan consonance et résonnance. Il y a de l'Un !, se plaît-il à énoncer, pour souligner la découverte d'Héraclite. Une telle exclamation écarte toute interprétation inspirée de l'Un parménidien. Il y a de l'Un!: la formulation nous indique que l'Un fait à chaque fois effraction dans le langage. S'il y a de l'Un, c'est que l'Un n'est pas là, une fois pour toutes, mais qu'il resurgit dans le logos humain, sous diverses formes, manifestant ainsi le pouvoir de liaison d'éros dont le propre est de faire un (En) d'une multitude immense (Panta) : «Y a de l'Un, c'est de cet énoncé que j'ai supporté mon discours l'année dernière, et certes pas pour confluer dans cette confusion originelle, car le désir ne nous conduit qu'à la visée de la faille où se démontre que l'Un ne tient qu'à l'essence du signifiant. Si j'ai interrogé Frege au départ, c'est pour tenter de démontrer la béance qu'il y a de cet Un à quelque chose qui tient à l'être, et, derrière l'être, à la jouissance " ${ }^{61}$.

70 C'est dire que l'Un est un signifiant, mais pas n'importe lequel. Il est le pouvoir signifiant du Logos lui-même : la loi même de tout signifiant ${ }^{62}$, le signifiant originel manquant (SO) parce que mis en abîme par les effets signifiants qu'il induit: «Le signifiant Un n'est pas un signifiant quelconque. Il est l'ordre signifiant en tant qu'il s'instaure de l'enveloppement par où toute la chaîne subsiste " ${ }^{63}$. C'est ce signifiant manquant qui émerge de la question, que semble récupérer le transfert, mais qui, faisant défaut dans la psychose, "laisse gésir» (liegen lassen), mais, de façon irrémédiable et pour de bon, un inconscient qui " ne fonctionne plus ", car lui manque le signifiant manquant ${ }^{64}$. Cette unité primordiale et immémoriale est celle de la présence ouverte par la parole. L'oracle dont parle Héraclite (fragment 93) en illustre, selon Lacan, la prosopopée : il n'avoue (legei) ni ne cache, mais il signifie ${ }^{65}$. Il est le Logos comme tel, qui «introduit deux ordres dans le monde, la vérité (alêtheia) et l'événement (Ereignis) » ${ }^{66}$. Mais, on s'en doute, cette présence est éprouvée comme absence, comme béance. Elle est en répit et en repos dans l'inconscient.

Revenons à la traduction de Lacan : Logos est "le lit où se lit ce qui s'élit ». On peut le deviner, le lit, c'est l'inconscient assimilé à l'hypokheimenon qui signifie, au sens littéral, le « ci-gît » et ne peut, par conséquent, être interprété en termes de substance. Il est le milieu du lais ou du legs, ces deux mots-clefs qui nous renvoient à l'expérience d'une donation et d'une transmission. D'une donation, car l'inconscient, selon une étymologie commune, laisse être et fait être (lassen) : il se présente comme le « lais ", car il « met-àreposer » le Logos, le fait être et naître comme un langage, mais aussi le laisse être, car, à peine né, il découvre l'espace d'où le Logos s'est retiré, le lais désignant aussi la grève découverte par le reflux des eaux. Le Logos passe dans l'inconscient selon des modes de rassemblement jamais stables, selon des configurations et des compositions mouvantes. Le Logos fait le lit de l'inconscient en s'y déposant et en s'y soustrayant. Si le lais est aussi est le legs, l'inconscient est aussi le lieu d'une transmission : le legs est, comme on 
le sait, une disposition gratuite qui n'implique ni mutualité ni réciprocité, puisqu'elle se trouve scellée et celée par le seul donateur dans l'écriture d'un testament.

On peut voir dans le terme philosophique hypokeimenon, comme aussi dans les métaphores du lais et du legs, le profil de la notion de structure, telle que Lacan l'applique à l'inconscient. Le langage est, en effet, pris dans une structure qui lui impose un ordre, mais il ne cesse de se modifier à la fois à partir de sa structure et à l'encontre de sa structure. À la différence de la catégorie qui fige le sens, la structure qui diffère d'elle-même le fixe et le refixe. Ce qui la constitue, en effet, ce ne sont pas des éléments, mais les relations qui, existant entre ses divers éléments, la produisent comme un ensemble métastable ou covariant. Ce sont les signifiants qui font "bouger " la structure langagière et c'est la structure qui leur impose un "ordre" de marche. C'est en elle que les signifiants se font et se défont, mais aussi se " fondent ", au double sens du terme. À la différence du psychiatre qui recourt plus volontiers aux catégories nosographiques, le psychanalyste se met à l'écoute des structures mobiles et labiles de l'inconscient qui rassemblent les signifiants en dérive et les mettent à reposer dans l'inconscient.

Lacan considère l'hypokeimenon, l'inconscient, comme étant le lieu de «l'inscription de la tension substantielle entre le symbolique et réel ${ }^{67}$. Or, une telle tension provient de ce que les structures qui le composent et le recomposent sont commandées par une écriture incessante du «signifiant qui représente le sujet pour un autre signifiant ». Mais, à ce niveau, l'Un surgit rétroactivement et de manière ponctuelle, dès lors que cette représentation se produit et que le signifiant fait signe $d u$ sujet. Ainsi, l'Un est-il la trace d'un passage : celui du sujet qui advient comme effet du signifiant : « La structure, lit-on dans l'Étourdit, c'est l'asphérique recelé dans l'articulation langagière en tant qu'un effet du sujet s'en saisit ${ }^{68}$. C'est cet effet qui fait structure. On se tromperait à identifier hypokeimenon et sujet, mais on ne peut penser le sujet sans le référer à l'hypokeimenon dont il joue, tel un chef d'orchestre, la partition.

\section{La lettre et lalangue}

Le Logos se marque et se remarque dans l'inconscient, au rythme d'un double geste de donation et de transmission, de "trans-diction", pour employer un mot de Lacan ou, ce qui revient au même, de transcription : comme un langage et comme une écriture. L'hypokeimenon, c'est le là du Da-sein, dira Heidegger. C'est, selon Lacan ce « là ", où ça parle et ou çà s'écrit. Il fait le lit de ce qui se lit : s'offre à la lecture, c'est-à-dire à la venue de l'écriture dans le langage. Mais le Logos est le lit qui se lit et s'élit. Le choix du mot «s'élit » n'est seulement pas guidé par le jeu de l'allitération, car il s'inscrit dans la chaîne des signifiants précédents comme un point de chute, un point d'orgue, un point de sustentation. C'est bien le Logos qui s'élit : c'est lui qui se présente, se rassemble et se met à reposer. L'inconscient est son terrain d'élection et de prédilection : il s'y élit domicile, à l'instar de la moisson que l'on met à l'abri. Le «ci-gît» est le gîte, note Heidegger dans son commentaire, et, plus tard, Dans acheminement vers la parole (Unterwegs zur Sprache), il en fait le site de la dite (Sage), la maison du Logos ${ }^{69}$. Mais c'est aussi le Logos qui s'é-lit: qui se donne à lire en s'élidant dans la Lettre, en s'y excrivant. L'hypokeimenon est à la fois l'Un et le Tout, le lieu du rassemblement des signifiants et de leur déclinaison: le lieu du trésor des signifiants qui émergent et émargent de cette élection. 
Lalangue où le logos se laisse « ouïr » une interprétation des deux sens fondamentaux dont Heidegger crédite le Logos : celui de rassembler ou de collecter et celui de lire ou de dire. Or, c'est l'Autre qui est le trésor des signifiants et, à ce titre, il est aussi le lieu du manque qui constitue le sujet. Comment comprendre cette double assignation? Si nous réfléchissons au processus de l'énonciation, nous constatons d'abord que les mots en appellent aux mots, que les signifiants en appellent aux signifiants. Telle est bien la preuve que la parole procède d'un manque : celui de ne pouvoir atteindre l'objet. Tout se passe comme si elle courait après le réel que son avènement avait ré-légué ou, ce qui revient au même, comme si elle faisait danser les mots sur le réel perdu pour en célébrer la sépulture, se persuadant ainsi qu'elle en consomme le deuil, car c'est bien elle, la parole, qui l'a enseveli et qui, chaque jour, en appelle à sa résurrection. La voici qui creuse ce vide irrémédiable en l'Autre, effet rétrospectivement éprouvé de ce que manquent les signifiants et qui se décline dans les différences, dans les interstices qui les séparent des uns des autres.

C'est cette dérive signifiante que prend en charge le sujet. Le voici surgir de l'acquiescement au Logos, dès lors qu'il se décide à être l'otage de la parole, de laquelle il est désormais condamné à se soucier, qui est son souci, parce qu'elle lui manque et qu'il la manque. On comprend à quel point est important le soin de parler ou d'écrire : le soin apaise, pour un temps, l'inquiétude consubstantielle au souci.

Le " trésor des signifiants " recouvre le code, où le sujet vient glaner les signifiants pour se représenter à travers eux. Mais «lalangue », quant à elle, désigne l'équipement en signifiants qu'il s'est approprié pour en faire sa langue, l'idiome de son désir : langue maternelle qui reprend les phonèmes provenant de cette première figuration de l'Autre. Elle est un savoir constitué d'affects : «Lalangue nous affecte d'abord par tout ce qu'elle comporte d'effets qui sont des affects " ${ }^{70}$, elle est, d'une certaine façon, le pathos du Logos et, note plus tard Lacan, en reprenant les mots de sa traduction : «Ce savoir, en tant que c'est dans le gîte de lalangue qu'il repose, veut dire l'inconscient ${ }^{71}$.Toujours est-il que le savoir de lalangue réunit des éléments disparates qui seront la matière première que le langage va reprendre et réorganiser en chaînes signifiantes : «Cet Un incarné dans lalangue est quelque chose qui reste indécis entre le phonème, le mot, la phrase, voire toute la pensée ${ }^{72}$.

\section{La Lettre où il s'élit et se lie}

Opérant l'articulation entre « le trésor des signifiants » et « lalangue », il y a la Lettre, c'està-dire les premiers dépôts de ces signifiants, qui, pour revenir à la terminologie freudienne, rassemblent les premiers écrits (Niederschriften) ou les premiers signes perceptifs (Wahnehmungszeichen) et constituent la couche lisible d'un réel abyssal où se croisent la mort et la vie, l'amour et la haine, le rapport sexuel et la jouissance qui résulte de l'impossible ratification de ce dernier. Lacan reprend cette notion à Freud, mais il l'interprète, notamment à la lumière de la théorie des ensembles de $\mathrm{N}$. Bourbaki qui utilise une lettre pour caractériser un assemblage donné, mais il précise aussitôt que la fonction de la lettre est de faire l'assemblage et non seulement de le désigner ${ }^{73}$. On dira que la Lettre, une et multiple, est l'écriture première qui colle au réel 
et en décolle et, ce faisant, en réalise la paraphrase symbolique. "Effet du langage " ${ }^{74}$, elle opère les premières fixations du symbolique, mais elle n'est pas pour autant à jamais figée dans sa matérialité, car elle est de nature topologique et non typologique : elle est «ce qui ne cesse pas de ne pas s'écrire " ${ }^{75}$. Elle est le signifiant de l'Un qui conjugue la nécessité et l'impossibilité : la nécessité de s'écrire et l'impossibilité de tout dire. Et la langue, de son côté, est la caisse de résonance de la Lettre, sa mise en musique, sa transcription affective.

Tel est le Logos : ce qui-s'étend-devant se déploie comme le trésor des signifiants (legen) qui, offerts à la moisson (lesen), se donnent à lire (lesen) dans la Lettre et à dire à partir de "lalangue ». Mais le trésor des signifiants étant ce que Lacan nomme l'Autre, on ne peut éluder la question de savoir si l'Un, c'est l'Autre ou si l'Un est différent de l'Autre. On se rapprochera de la juste pensée de Lacan en disant, mais sans en écarter la connotation sexuelle, que l'Un, c'est l'Un en l'Autre ou, plus exactement, le trait qui barre l'Autre pour s'y inscrire. C'est ce trait, cette barre qui est, pour ainsi dire, la première Lettre : «Car l'Autre.... C'est l'Un-en-moins " ${ }^{76}$. C'est en émergeant dans ce rapport à l'Autre que l'Un est l'Un : ce qui fait que le savoir tient tout de son rapport à cet Autre barré qu'est la vérité et ce qui fait aussi qu'il ne saurait jamais en avoir raison. Ce trait, qui, en faisant «barrage» - Heidegger nous rappelle que «Legi » en alémanique signifie le barrage du torrent -, marque le croisement de l'Un et l'Autre, est constitutif du Logos dont la présence est absence et le dévoilement dissimulation. Il opère la partition originelle entre le moins et le plus : l'Autre évide l'Un et le présente comme moins-un, tandis que l'Un limite l'Autre et le présente comme un plus, un plusde-jouir, souligne Lacan, comme ce «jenes mehr » qui, selon Heidegger, tisse le fil rouge de la moisson. Toujours est-il que l'Un, comme croisement de l'Un et de l'Autre, trace le chemin de la mesure qui excède le manque et qui creuse l'excès.

\section{Les anamorphoses du Logos}

L'inconscient, comme legs ou comme lais, est structuré par la Lettre qui est la partition que joue lalangue. La Lettre, c'est l'écriture du réel perdu, sa marque indélébile : ce qui du réel s'écrit. On pourrait dire qu'elle est la marque du refoulement. Quant à lalangue, elle fait parler la Lettre. Mais, pour passer de la Lettre à lalangue, il convient de lire la Lettre et de l'ouïr de lalangue. Nous avons à faire à trois régimes qui se recoupent : celui de la constitution de la Lettre qui s'élit dans l'inconscient, celui de la lecture de ce qui s'y lit, enfin celui de l'écoute dans lalangue de ce qui s'y ouït. Quant au langage, il est l'élocution (lexis) de cette lecture et de cette écoute. Un tel parcours est celui de la genèse du Logos comme Un et Tout. Comme Un, car il se rassemble dans la Lettre, puis dans lalangue et enfin dans la lexis. Comme Tout, car l'Un émerge d'un enchaînement de différences : celle du passage de l'élection originelle à son inscription dans la Lettre, celle du passage de la Lettre à lalangue, celle, enfin, du passage d'un signifiant à l'autre (S1----S2-----) qui réalise le maillage de la lexis. L'Un est le signifiant manquant (so) qui, au fil de ses anamorphoses, fait et défait les différences qui prennent indéfiniment la mesure d'un Tout hypothétique.

La Lettre est une et plurielle, puisqu'elle condense les premiers enchaînements signifiants relatifs à la vie, à l'amour, à la mort, à la sexualité. Lalangue qui la transpose dans le registre du langage est elle-même une et plurielle et c'est pour marquer ce rassemblement offert à l'écoute que Lacan ne sépare pas l'article du substantif. Quant au langage locutoire, il obéit à la même logique de rassemblement qui relève de la 
dialectique de l'un et du multiple. Telles sont les anamorphoses du Logos qui, à partir de son site primordial, se disperse et se reconfigure dans la Lettre, dans lalangue et dans le langage. À chacun de ces niveaux joue la dialectique de l'Un et du Tout.

\section{Homologuer le legs : l'admission primordiale (Die Bejahung)} lalangue, au lire et à l'ouïr des mortels, qui se réalisent dans l'homologein. Ouïr le Logos, c'est s'accorder à sa voix silencieuse, pour ensuite la traduire en mots, la lire et la dire. Cette écoute fait elle-même partie du Logos lui-même : Heidegger souligne qu'il ne peut $\mathrm{y}$ avoir de moisson sans le travail des moissonneurs qui glanent, trient et collectent les fruits de la récolte, pour ensuite les rassembler et les mettre à l'abri. Elle se réalise, selon Lacan, dans l'ouïr de l'inconscient, où s'élit et se lit le Logos. La parole vraie est celle qui témoigne de cette écoute. On peut interpréter le célèbre « Là où c'était, je dois advenir » comme étant le tracé du chemin de l'homologein : « Là où çà s'élit et se lit, là où çà parle, là où çà gît, je me dois de lire et de dire ".

On peut, par conséquent, interpréter l'homologein, dans le champ de la psychanalyse, d'une double manière. Le dire et le parler quotidiens expriment la manière dont nous accordons à l'un/in-conscient, dont nous opérons la partition et le partage des signifiants dont il est le trésor, dont nous les moissonnons et dont nous les enchaînons sous les divers modes du transfert, du refoulement, du rejet, de la dénégation ou de la sublimation, autant de termes que Lacan convie dans sa traduction. Nul n'est en accord avec lui-même s'il n'écoute pas le Logos qui parle en lui. Au fur et à mesure que nous parlons, nous refaisons le travail de la moisson, nous réaménageons le gîte où nous l'engrangeons, nous reconstituons indéfiniment le ci-git, le « là où c'était ».

Mais il y a plus: on ne saurait rester sourd à l'impératif éthique qui est celé dans l'homologein. Le Logos est mandat et le mortel est mandaté à le cueillir et l'accueillir. Le mortel qui parle contracte une dette originelle envers le Logos et cette dette, il se doit de l'honorer en s'accordant à lui. On le voit, Lacan formule l'interprétation heideggérienne de l'accord réalisé par l'homologein dans les termes de la Bejagung freudienne qui recouvre l'acquiescement à l'Autre:«La Bejahung n'est autre que la condition primordiale pour que du réel quelque chose vienne à s'offrir à la révélation de l'être ou, pour employer le langage de Heidegger, soit laissé être. Car c'est bien à ce point reculé que Freud nous porte puisque ce n'est que par après que quoique ce soit puisse y être retrouvé comme étant $"{ }^{77}$.

Il faut donc admettre, à la source des processus langagiers, « une Bejagung primordiale, une admission dans le sens du symbolique, qui elle-même peut faire défaut ${ }^{78}$. Si l'on veut expliciter le mode opératoire de cet homologein, il nous faut distinguer l'Un comme signifiant originel du Logos (S0) et l'Un conçu comme le signifiant maître (S1) qui scelle l'accord de l'homologein. L'Un y surgit de la barre, du barrage, qui marque les limites de l'accès au Logos conçu comme le discours de l'Autre, mais cette barre est inséparablement le trait, par lequel se réalise l'identification du sujet de l'inconscient. Si le désir est désir de l'Autre, c'est parce que l'Autre en appelle au désir du sujet : il est le lieu de la reconnaissance du désir, mais aussi, dans la mesure où il ouvre à la rencontre d'autrui, le lieu du désir de reconnaissance. On le comprend, l'homologein est le oui/l'ouie de l'ouïr, qui, en ouvrant à l'inconscient l'accès au symbolique, est un "jouir ", car il fait naitre le désir du sujet en l'articulant à l'oür et, par voie de conséquence, permet à ce 
dernier de s'entendre avec autrui. Il faut ajouter que le signifiant maître qui produit cet embrayage initial sur le Logos va, note Lacan, se déployer en un essaim signifiant et bourdonnant: S1-----S2----S3 : "L'S1, l'essaim, signifiant maître, est ce qui assure la copulation du sujet avec le savoir " ${ }^{79}$. Il y a un S Un.

Mais si l'homologein exprime l'exigence de chaque sujet de se mettre en accord avec l'inconscient, qui fait le lit du Logos, il ouvre, du même coup, l'espace d'une écoute possible de l'inconscient d'autrui, c'est-à-dire de la manière dont autrui peut témoigner de l'accord qu'il réalise lui-même. Le Logos est le lieu où les mortels peuvent s'entendre entre eux : il rend compte certes de la singularité de lalangue, mais il déploie le langage comme milieu de la communication des idiomes. Il est l'Un en étant l'Autre : figure du tiers qui unit et sépare. C'est dans l'homologein, dans l'ouïr fondamental du Logos qui s'élit dans l'inconscient, s'y offrant à dire et à lire, que peut avoir lieu l'écoute analytique. C'est de la réception qu'il a fait du Logos, de la manière dont il l'a entendu, du tour de moisson qu'il a réalisé et sauvegardé dans son inconscient que le sujet rend conte/compte. Le psychanalyste, quant à lui, répète le geste de la moisson qui, dans l'acte d'interprétation, collecte, sélectionne et sauvegarde les signifiants du dire, les "met-à-reposer ", pour que l'analysant puisse, dans le lit du transfert, les lire et les lier, d'une manière qui lève l'inouï, en reprenant le geste du moissonneur. Pour reprendre les termes de Heidegger, l'écoute analytique, à l'instar de celle que nous faisons de l'Un pluriel d'Héraclite, est l'accueil de ce que l'on cueille: le terme allemand "Annahme " signifie accueil et hypothèse. C'est la succession des hypothèses interprétatives qui font le lit de cette insolite écoute.

\section{Mise en abyme et émergence de l'un : la chaîne des signifiants}

87 Lacan marque, dans sa traduction, la relation dialectique existant entre l'Un et Toutes choses. L'Un, c'est le trait signifiant qui, en s'effaçant, fait surgir la chose dans la présence. Mais ce trait signifiant n'a de sens qu'en relation avec d'autres traits signifiants qui font glisser la chose vers autre chose. En clair, on peut dire que la chaîne des signifiants est une succession des « uns » qui, en se substituant les uns aux autres, ne cesse de renvoyer à autre chose qui constitue l'horizon de la chose présentée : «Le «En Panta» laisse se présenter ensemble dans l'être d'une présence, ce qui est essentiellement divergent l'un de l'autre et s'oppose ainsi l'un à l'autre par une absence contrariée... Ce qui est ainsi séparé en une trajectoire qui du lointain le plus extrême fait le départ entre la présence et l'absence, diapheromenon, le lais où se lit ce qui s'élit le laisse se présenter dans sa rétribution ${ }^{80}$. On peut dire que l'Un émerge, à chaque fois, de la logique discontinue des signifiants qui se succèdent les uns aux autres et enserrent potentiellement Toutes choses. L'émergence du trait signifiant tient lieu de la chose et son retrait laisse place à un autre trait qui tient lieu d'autre chose. L'Un, c'est l'éclair héraclitéen de la présence qui rassemble, en un instant, les signifiants épars des choses disparues.

88 C'est bien, en effet, le rapport à cet Autre qu'est l'Un qui dessine le milieu de la rencontre de toutes choses (panta) : en l'occurrence, des signifiants qui, en disant les choses, en les faisant naître au langage, renvoient, de manière oblique, au sujet, au cigît supposé, que le signifiant représente pour un autre signifiant. Il ne s'agit pas d'identifier l'hypokeimenon au sujet réflexif, mais il s'agit bien d'y voir le profil de l'inconscient d'un sujet supposé. La critique de la subjectivité par Heidegger ouvre le 
chemin pour repenser le sujet comme être questionnant: comme étant «ce qui ? énigmatique " qui se trouve être mis en question par le Mandat du Logos, comme ce mortel (Dasein) qu'il est, mais qui, à chaque fois, fait sienne cette question de façon singulière (Jemeinigkeit). Ainsi, le sujet préposé au Logos est-il toujours supposé : non seulement parce qu'il n'existe que comme questionnant et questionné, mais aussi parce que c'est en répétant cette question pour la faire sienne qu'il existe "à chaque fois " comme sujet supposé du Logos. On peut ainsi entendre la parole d'Héraclite comme témoignant de la position de l'inconscient dans le rapport à l'Autre : au Logos qui, dans l'homologein, ouvre le milieu du rassemblement et de la dissémination des signifiants et qui dessine en pointillé la matrice de la subjectivité.

La dialectique héraclitéenne de l'Un et du Tout se trouve ainsi relue dans l'espace de la négativité et de la subjectivité. Car le Logos, c'est bien l'Un de la béance ou de la faille qui glisse sous la parole, mais c'est aussi l'ensemble des discours qui s'y rattachent. On a de la peine à distinguer en français le dire et le dit qui se confondent dans l'énonciation, alors que, en allemand, l'énonciation (Aussage) est distinguée de son contenu (das Ausgesagte). Mais laissons de côté cette particularité linguistique, pour mettre en lumière l'articulation que le Logos opère entre la présence et les choses présentes et entre la parole qui ouvre à la présence et les énoncés qui, dans son prolongement, étendent cette présence à l'ensemble des choses. On perçoit alors plus clairement que l'Un est ce qui dans le Logos exprime, après coup, la marque du rapport à l'Autre, trait d'un renvoi qui survient, sur le mode rétroactif, pour combler le retrait du sens, et qui est aussi et inséparablement le trait unaire, par lequel le sujet se rassemble en faisant effraction dans la chaîne signifiante.

Il y a de l'Un, parce que le sujet soutient le Logos qui le soutient : c'est, en effet, le sujet qui, en survenant dans la parole, déplace la marque originelle de l'aliénation, en l'inscrivant dans le mouvement de l'écriture d'uns successifs. Le schéma lacanien de l'enchaînement des signifiants S1------S2 qui prend la mesure de l'extension du Logos, nous montre que ce que l'on appelle la béance marque la limite de l'impossible saisie de l'origine, l'écart d'une différence qui se remarque à chaque glissement de signifiant et la ligne de l'horizon de l'absence du signifiant ultime. Le sujet se dit dans cette différence que, par le trait unaire, il délimite en la marquant en amont et en aval. L'Un exprime paradoxalement la démesure du Logos emporté par le retrait hyperbolique de la différence qui le constitue et la mesure que lui impose, sur le mode de la répétition, le trait symbolique marqué par l'émergence du sujet.

On peut, en suivant le fil du commentaire de Heidegger, prolonger cette interprétation psychanalytique du En/Panta à la lumière de la vérité conçue comme dévoilement. Le Logos se présente, en effet, comme le lieu de la vérité, en mettant en tension les deux pôles qui le constituent. En tant que Unique, il est le Sens, c'est-à-dire l'Être-à-être, l'Être qui s'élit à la présence, comme l'indiquent les métaphores du lais, du legs et du mandat. Mais il survient à la présence en se phénoménalisant dans la présentation qu'il fait de toutes choses: celle de l'être de l'étant, de l'être comme tel. L'Unique «met-àreposer » toutes choses. On peut aisément le comprendre : le Logos, comme Être-à-être, comme Sens, détient la préséance ou la prééminence qui est celle de la mise en présence : il précède et excède l'ensemble des choses qu'il phénoménalise. À l'inverse, en tant qu'il est la totalité des choses qu'il phénoménalise dans la disparité, il dissimule le lit de la présence où il les «met-à-reposer ». D'une certaine façon, la présence se cache dans la présentation qu'elle réalise. Voilà pourquoi c'est du Logos même que l'on 
peut penser la vérité comme le voilement de la présence. Tout se passe comme si l'Êtreà-être ne réalisait son Mandat qu'en se retirant de la présence qu'il commande. L'Un, c'est le pointage sémantique de la vérité qui advient à la présence comme Logos et, qui, en se voilant, fait apparaître Toutes choses ${ }^{81}$.

Heidegger pense le Logos comme la révélation du Sens qui ne cesse de se voiler dans sa présentation même : comme l'a-lêtheia qui surgit du Léthè/Lais-thè et y retourne. Il est, à ce titre, pensé comme l'ouverture d'une spatialité originaire dont le temps marque le tempo des apparitions et des disparitions. Le Logos est le lieu originaire de l'espace et du temps. Telle est la raison, pour laquelle Heidegger l'assimile, dans le commentaire qu'il consacre à la Physique d'Aristote, à la Physis qui est précisément le milieu de la genèse de l'espace et du temps, le temps mesurant les tempos de l'ouverture et de la fermeture de l'espace ${ }^{82}$. Mais si le Logos est Physis, c'est aussi reconnaître du même coup, qu'il est nature, matérialité, vie. La chair du Logos prend corps dans la parole.

Or, c'est bien le rapport du Logos à la Vérité perçue comme dévoilement qui intéresse au plus haut point la psychanalyse. Certes, cette lecture déplace le sens sur le champ de l'inconscient et, par conséquent, de la subjectivité singulière. Mais le transfert auquel elle oblige ne contredit pas pour autant les thèses du commentaire de Heidegger. Même si le Logos s'invertit en Mythos dans l'inconscient, il y conserve sa duplicité, il reste le lieu de la présence : de l'Être à dire et à lire, du Sens à énoncer et des effets de sens qui le voilent en le dévoilant; de l'Autre pensé en termes d'une spatialité ouverte par le jeu de ses ouvertures et de ses fermetures et d'une temporalité rythmée par les pulsations qu'elle génère ; d'une énonciation indéfinie, sans cesse soufflée par l'énoncé. Le legs s'y trouve délégué sous la double forme de l'allégation et de la relégation, pour reprendre les termes ingénieux de la traduction.

\section{Zeus ou le nom du père}

94 Il nous faut, pour terminer, reprendre, sous un autre jour, le commentaire de Heidegger concernant la possibilité ou l'impossibilité que l'Un puisse être dénommé du nom de Zeus. Même si l'analyse que fait Heidegger de l'évocation du nom de Zeus, ne va pas au fond des choses, elle présente au traducteur tous les éléments d'une ultime interprétation possible. En rapprochant de ce fragment deux autres fragments, elle rappelle que l'éclair, emblème de Zeus, gouverne tout le présent dans la présence (B 64) et que l'Un ne veut pas et pourtant veut qu'on l'appelle Zeus (B 32). Elle montre, en effet, que le refus et la revendication de cette dénomination religieuse ne sont pas contradictoires, parce que les deux énoncés n'envisagent pas le Logos sous le même rapport. S'il est l'Unique, il répugne à toute dénomination, parce qu'il n'est réductible à aucune figure. Mais s'il est l'ensemble des choses présentes, il peut prétendre à une telle dénomination, car le nom de Zeus a pour effet de servir de relais symbolique entre la totalité des choses présentes et l'unicité de l'Un. Pour recourir à la théorie kantienne, disons que cette dénomination est schématisée, en faisant appel au gouvernement du plus haut des Dieux, par l'éclair fulgurant provenant de la survenue de la présence et de son retrait au sein de la diversité des choses présentes.

Mais la traduction de Lacan pousse le commentaire de Heidegger dans ses derniers retranchements. La dénomination religieuse est une « sublimation » qui se produit sur le mode d'une " dénégation ". La sublimation entraîne le mortel à faire l'expérience de la grâce de la présence à partir de ce «Trait » caractéristique de son être qu'est le 
langage. Quant à la dénégation, elle légitime le double langage, selon lequel l'ensemble des choses présentes (En Panta) contient un "cillement d'ouverture sur ce qu'est le Logos " ${ }^{83}$ ou encore, peut-on lire plus loin, « un cillement d'ouverture sur la divinité de Dieu $"{ }^{84}$. Elle nous renvoie à l'univers des signes (Wincke) et au monde des signifiants. Elle invite à interpréter la causalité de l'Être suprême en termes de fonction symbolique et de fonction paternelle, car le dieu suprême est aussi le père des dieux. Telle est l'échappée qui se profile à l'horizon du commentaire de Heidegger. Zeus est la métaphore de la fonction paternelle, clef de voûte de la fonction symbolique. Il nous enseigne que le Logos nous permet d'identifier les choses dans leur unité et leur disparité. C'est la fonction paternelle qui unifie et rassemble toutes choses et qui se réalise dans l'homologein, autrement dit dans la correspondance du mortel au Logos qui parle en lui. C'est elle qui, parce qu'elle est le relais entre l'Un et le Tout, entre les deux pôles du Logos, rassemble, c'est-à-dire structure et finalise l'ensemble des choses et, à un titre privilégié, le dire humain. La fonction paternelle est la loi secrète du lais et du legs: elle délègue la capacité d'alléguer et de reléguer. Ce n'est pas pour rien que c'est autour du signifiant "loi ", signifiant manquant dans le texte, que Lacan, devenu l'analyste de Heidegger, fait graviter les signifiants déterminants de sa traduction : signifiant visé, mais jamais énoncé comme tel, signifiant qui, parce relégué, en est encore plus éloquent. La fonction paternelle enracine le monde symbolique dans l'essence hyperbolique du Logos. Comme Zeus, elle gouverne, commande et ordonne, mais elle tient du Logos la grâce d'être la loi qui lie et délie les choses présentes.

On le voit, l'interprétation de Lacan éclaire les présupposés ontologiques de la pensée de Heidegger et ne renie pas sa critique de la subjectivité. Elle s'attache à l'explicitation $\mathrm{du}$ «legein» du Logos et à la vérité (a-lêtheia) qui est son mode d'apparition. Ainsi, le commentaire de Heidegger est-il susceptible de lui fournir un cadre assez large, pour qu'il puisse y intégrer sa thèse centrale de l'inconscient structuré comme un langage et, ce qui est un paradoxe, sa conception de l'homme comme sujet. Il existe, en effet, un schématisme commun entre l'homme posé comme Dasein et l'homme conçu comme être désirant : c'est celui d'une existence divisée (ek-sistence), parce que traversée par le langage. Mais Lacan se met en retrait de la conception destinale du Logos qui compose les profils d'une histoire de l'Être. Sans doute n'entre-il pas dans la critique heideggérienne de la science et de la technique, dont il n'a pas eu à traduire les pages : mentionnée dans la première version, celle-ci n'est explicitée que dans la seconde version. Le destin des pulsions qui est relatif au devenir-sujet de l'homme n'a rien à voir avec le destin de l'Être qui transcende le désir de l'homme.

À juste titre, Lacan considère le texte qu'il nous livre comme un article. Car non seulement il nous fournit une traduction rigoureuse fidèle au lexique et à la syntaxe de la langue allemande, mais il s'emploie, à partir d'une recherche minutieuse d'équivalences, à nous faire entendre le motif symbolique du propos heideggérien. Ainsi, la traduction est-elle, du même coup, une interprétation qui n'a nul besoin d'être explicitée dans une herméneutique dont on sait bien ce que Lacan en pense. La traduction est elle-même un acte d'interprétation. À ce titre, Lacan, traducteurinterprète, se surprend à jouer à l'analyste de Heidegger. Rappelons, pour terminer, l'avertissement qu'il lui adresse et qui le renvoie, finalement, par-delà la métaphysique, à l'écoute du Logos : « Pour mon "ami” Heidegger évoqué plus haut du respect que je lui porte, qu'il veuille bien s'arrêter un instant, vœu que j'émets purement gratuit puisque je sais bien qu'il ne saurait le faire, s'arrêter, dis-je, sur cette idée que la métaphysique 
n'a jamais rien été et ne saurait se prolonger qu'à s'occuper de boucher le trou de la politique. C'est son ressort $\gg{ }^{85}$.

\section{NOTES}

1. J. LACAN, Les Écrits, Paris, Le Seuil, 1966, p. 288.

2. J. LACAN, Le Séminaire, Livre VII. L'éthique de la psychanalyse, Paris, Le Seuil, 1986, p. 321.

3. Trad. Lacan, p. 11. Les références à la traduction renvoient à la pagination du texte ci-dessus.

4. Trad. Préau, p. 249.

5. Trad. Lacan, p. 9.

6. Trad. Lacan, p. 11.

7. Texte Heidegger, p. 236.

8. Trad. Préau, p. 254.

9. Trad. Lacan, p. 12.

10. Trad. Lacan, p. 12.

11. Texte Heidegger, p. 216.

12. Trad. Lacan, p. 17.

13. Trad. Préau, p. 260.

14. Trad. Lacan, p. 11.

15. Texte Heidegger, p. 226.

16. Texte Heidegger, p. 226 ; trad. Lacan, p. 22.

17. Trad. Lacan, p. 18.

18. Op. cit., p. 13.

19. Op. cit., p. 11.

20. Op. cit., p. 13.

21. Op. cit., p. 14.

22. Op. cit, p. 16.

23. Texte Heidegger, p. 222.

24. Trad. Lacan, p. 17.

25. Ibid.

26. Op. cit, p. 15.

27. Op. cit, p. 16.

28. Op. cit., p. 21.

29. Texte Heidegger, p. 223.

30. Trad. Préau, p. 263.

31. Trad. Lacan, p. 19.

32. Texte Heidegger, p. 223.

33. Trad. Lacan, p. 18.

34. Op. cit., p. 14.

35. Op. cit., p. 13.

36. Texte Heidegger, p. 217.

37. Trad. Préau, p. 255.

38. Trad. Lacan, p. 13.

39. Op. cit., p. 14. 
40. Op. cit., p. 20.

41. Ibid.

42. Trad. Préau, p. 272, trad. Lacan, p. 24.

43. Texte Heidegger, p. 224.

44. Trad. Préau, p. 265 ; trad. Lacan, p. 20.

45. Texte Heidegger, p. 225.

46. Trad. Préau, p. 273 ; trad. Lacan, p. 24.

47. HEIDEGGER, « La parole d'Anaximandre », Chemins qui ne mènent nulle part, Paris, Gallimard, 1962, p. 299.

48. Trad. Lacan, p. 15.

49. Op. cit., p. 21.

50. Op. cit., p. 21.

51. Ibid.

52. Op. cit., p. 22.

53. Op. cit., p. 23.

54. Ibid.

55. Ibid.

56. Op. cit., p. 24

57. Ibid. Voir le texte allemand, p. 229 : „... die Schickungen des Geschicks”.

58. « Lettre sur l'humanisme » (1946), Questions III, trad. R. Munier, Paris, Gallimard, 1966, p. 101, $114,133$.

59. Trad. Lacan, p. 25.

60. J. LACAN, Le Séminaire, Livre XX, « Encore », Paris, Le Seuil, 1975, p. 32.

61. . J. LACAN, Encore, op. cit., p. 12.

62. J. LACAN, Le Séminaire, Livre VIII, « Le transfert », Paris, Le Seuil, 1991, p. 143 et 144.

63. J. LACAN, Encore, op. cit., p. 13.

64. J. LACAN, Le Séminaire, Livre III, « Les psychoses », Paris, Le Seuil, 1981, p. 143

65. J. LACAN, Encore, op. cit., p. 103.

66. J. LACAN, Le Séminaire, Livre VII, « L'éthique de la psychanalyse », Paris, Le Seuil, 1986, p. 308.

67. J. LACAN, Les psychoses, Op. cit., p. 371.

68. J. LACAN, « L'étourdit », Autres écrits, Paris, Le Seuil, 2001, p. 488.

69. M. HEIDEGGER, Acheminement vers la parole, trad. F. Fédier, Paris, Gallimard, 1976, p. 240 : « Ainsi ce qui se déploie dans la parole est la Dite (die Sage) en tant que monstre (die Zeige) ».

70. J. LACAN, Encore, op. cit., p. 127.

71. J. LACAN, Encore, op. cit., p. 129.

72. J. LACAN, Encore, op. cit., p. 131.

73. J. LACAN, Encore, op. cit., p. 46.

74. Ibid., p. 45.

75. Ibid., p. 55.

76. Ibid., p. 116.

77. J. LACAN, Écrits, Paris, Le Seuil, 1966, p. 388.

78. J. LACAN, Le Séminaire, Livre III, « Les psychoses », op. cit., p. 21.

79. J. LACAN, Encore, op. cit., p. 130 (voir aussi p. 13 et 127).

80. Trad. Lacan, p. 21.

81. Op. cit., p. 19-20. Voir à ce sujet : J.-L. NANCY, Le Sens du monde, Paris, Galilée, 1993, p. 25 s.

82. Voir HEIDEGGER, Die Physis bei Aristoteles, Frankfuhrt/a/ main, V. Klostermann, 1967.

83. Trad. Lacan, p. 20 et 24.

84. Trad. Lacan, p. 22.

85. J. LACAN, Autres écrits, Paris, Seuil, 2001, p. 554-555. 


\section{RÉSUMÉS}

La republication de la traduction qu'a faite J. Lacan de la conférence "Logos " de Martin Heidegger est riche d'enseignements. L'auteur du commentaire montre, dans une première partie, à quel point le traducteur a le souci de faire coller son texte à la lettre, non directement à coup de dénotations, mais par les effets signifiants produits par le jeu des connotations et des assonances. Il explicite, dans une seconde partie, les divers motifs de l'interprétation psychanalytique qui constitue l'horizon de la traduction et souligne combien ce texte auquel Lacan fait souvent allusion explicitement ou implicitement dans ses Séminaires constitue une référence fondamentale de sa pensée.

This commentary of Lacan's Heidegger translation examines first the translator's care in rendering the letter of the text and not just its spirit, wielding the effects in meanings produced by plays with connotations and assonances. In a second section, Resweber unpacks to make explicit the various patterns of the psychoanalytical interpretation that serve as a horizon to Lacan's translation, showing how much central this conference is within Lacan's own work.

Jean-Paul Resweber zeigt in einem langen Kommentar, wie die Übersetzung von Lacan original und sinnreich ist und wie sie auf eine psychoanalystische Auslegung beruht, die die Architektur des Denkens von Lacan gründet.

\section{AUTEUR}

\section{JEAN-PAUL RESWEBER}

Jean-Paul Resweber, fondateur avec Benoît Goetz de la revue Le Portique, a enseigné la philosophie à l'Université de Strasbourg (1969-1989), à l'Université de Bretagne Occidentale (1989-2001) et, à partir de 2002, à l'Université de Lorraine (Metz), dont il est désormais professeur émérite. Il a publié aux Éditions du Cerf et aux PUF divers ouvrages sur la question de l'herméneutique et de l'interprétation, sur l'interdisciplinarité et sur l'éducation. Il vient de publier en 2012 un ouvrage intitulé Questions de soin (L'Harmattan). 